\title{
Classical Mechanics and Geometric Quantization on an Infinite Dimensional Disc and Grassmannian
}

\author{
O.T.Turgut \\ Institut Mittag-Leffler \\ Auravägen 17, S-182 62, Djursholm, Sweden \\ and \\ Department of Physics \\ Bosphore University \\ Bebek, Istanbul, Turkey*
}

November 1998

\begin{abstract}
We discuss the classical mechanics on the Grassmannian and the Disc modeled on the ideal $\mathcal{L}^{(2, \infty)}$. We apply methods of geometric quantization to these systems. Their relation to a flat symplectic space is also discussed.
\end{abstract}

\section{Introduction}

We will analyze geometric quantization of a classical system which has as its phase space the infinite dimensional Grassmannian or the Disc modeled on the ideal $\mathcal{L}^{(2, \infty)}\left(\mathcal{H}_{+}, \mathcal{H}_{-}\right)$. There are two motivations for our work. The classical dynamics studied should correspond to the large- $N_{c}$ limit of a quantum system which requires a logarithmic renormalization. Its quantization should give us an understanding of this system in the Schrödinger picture. This picture has some advantages over the scattering matrix, as well-known in the physics literature. The second is to study and understand infinite dimensional systems, their quantization should lead to some interesting mathematical questions. A good example is typical two dimensional field theory models, which do not require a renormalization but only a normal ordering [20, 21]. It will be interesting to develop the necessary tools for more complicated systems, and perhaps give a more precise meaning to renormalized field theories. We should

*after March 99 
add that, in this article we do not study any particular Hamiltonian, and associated delicate domain problems. In some sense we have only made an attempt to study part of the kinematics. The full understanding will require studying a specific model.

\section{The Disc and the Grassmannian}

Our approach is inspired from the discussion of the Grassmannian in the book by Pressley and Segal [18] and closely follows our previous work [21]. We will extend some of our previous ideas to this case.

Let $\mathcal{H}$ be a separable infinite dimensional complex Hilbert space; $\mathcal{H}_{-}$and $\mathcal{H}_{+}$are two orthogonal isomorphic subspaces with $\mathcal{H}=\mathcal{H}_{-} \oplus \mathcal{H}_{+}$. Physically, one can think of these two spaces as the decomposition of the one particle Hilbert space into positive and negative energy states.

Define the Disc $D_{1+}\left(\mathcal{H}_{-}, \mathcal{H}_{+}\right)$to be the set of all operators $Z: \mathcal{H}_{+} \rightarrow \mathcal{H}_{-}$such that $1-Z^{\dagger} Z>0$ and $Z$ is in $\mathcal{L}^{(2, \infty)}\left(\mathcal{H}_{+}, \mathcal{H}_{-}\right)$. We refer to the Appendix for the ideals $\mathcal{L}^{(2, \infty)}$ and $\mathcal{L}^{(1, \infty)}$.

Since the space $\mathcal{L}^{(2, \infty)}\left(\mathcal{H}_{+}, \mathcal{H}_{-}\right)$is contractible, the set of $Z$ for the Disc can be taken as a coordinate system. It is an infinite dimensional complex manifold, modeled on a Banach space.

In a similar spirit, we define the Grassmannian to be the set of closed subspaces $W$ of $\mathcal{H}$, such that the projection $\operatorname{Pr}_{+}: W \rightarrow \mathcal{H}_{+}$is Fredholm and the projection $\operatorname{Pr}_{-}: W \rightarrow \mathcal{H}_{-}$is in $\mathcal{L}^{(2, \infty)}\left(W, \mathcal{H}_{-}\right)$. We first define natural group actions on these spaces. These group actions are used to prove that these are manifolds!

We introduce the following pseudo-unitary group which is a subset of the invertible operators from $\mathcal{H}$ to $\mathcal{H}$ :

$$
U_{1+}\left(\mathcal{H}_{-}, \mathcal{H}_{+}\right)=\left\{g \mid g \epsilon g^{\dagger}=\epsilon, \quad g^{-1} \text { exists and }[\epsilon, g] \in \mathcal{L}^{(2, \infty)}\right\} .
$$

Here $\epsilon=\left(\begin{array}{cc}-1 & 0 \\ 0 & 1\end{array}\right)$ with respect to the decomposition $\mathcal{H}=\mathcal{H}_{-} \oplus \mathcal{H}_{+}$. If we decompose the matrix into block forms,

$$
g=\left(\begin{array}{ll}
a & b \\
c & d
\end{array}\right)
$$

we have, $a: \mathcal{H}_{-} \rightarrow \mathcal{H}_{-}, b: \mathcal{H}_{+} \rightarrow \mathcal{H}_{-}, c: \mathcal{H}_{-} \rightarrow \mathcal{H}_{+}$and $d: \mathcal{H}_{+} \rightarrow \mathcal{H}_{+}$. Then, the off-diagonal elements $b$ and $c$ are in $\mathcal{L}^{(2, \infty)}$ and the diagonal elements $a$ and $d$ are bounded operators. In fact they are invertible operators, since their spectrum does not contain zero. The conditions on the off-diagonal elements imply some control over how much $\mathcal{H}_{+}$and $\mathcal{H}_{-}$ mix with each others.

\footnotetext{
[9].

${ }^{1}$ generalizations of this kind has been pointed out in [18], and explored in 17 for the ideals $\mathcal{L}^{p}$. See also
} 
We define an action of $U_{1+}\left(\mathcal{H}_{-}, \mathcal{H}_{+}\right)$on the Disc $D_{1+}$ :

$$
Z \mapsto g \circ Z=(a Z+b)(c Z+d)^{-1} .
$$

The condition $1-Z^{\dagger} Z>0$ implies that $c Z+d$ is invertible and bounded. Since the space of $\mathcal{L}^{(2, \infty)}$ is a two-sided ideal, $(a Z+b)(c Z+d)^{-1}$ is still in $\mathcal{L}^{(2, \infty)}$. Thus our action is well-defined.

The stability subgroup of the point $Z=0$ is $U\left(\mathcal{H}_{-}\right) \times U\left(\mathcal{H}_{+}\right), U\left(\mathcal{H}_{ \pm}\right)$being the group of all unitary operators on $\mathcal{H}_{ \pm}$. Moreover, any point $Z$ is the image of 0 under the action of the group, $g \circ(Z=0)=b d^{-1}$. (note that $b d^{-1}$ is in $\mathcal{L}^{(2, \infty)}$ and $d^{\dagger} d=1+b^{\dagger} b$ implies that $\left.1-\left(b d^{-1}\right)^{\dagger} b d^{-1}>0\right)$. We therefore see that $D_{1+}$ is a homogeneous space and given by the quotient;

$$
D_{1+}=U_{1+}\left(\mathcal{H}_{-}, \mathcal{H}_{+}\right) / U\left(\mathcal{H}_{-}\right) \times U\left(\mathcal{H}_{+}\right) .
$$

It is possible to view $G r_{1+}$ as a coset space of complex Lie groups. Incidentally this will define a complex structure on $G r_{1+}$ which will be useful for geometric quantization. Define a subset of the general linear group

$$
G L_{1+}=\left\{\gamma \mid \gamma \text { is invertible; }[\epsilon, \gamma] \in \mathcal{L}^{(2, \infty)}\right\}
$$

When we decompose g into $2 \times 2$ submatrices $\gamma_{12}, \gamma_{21} \in \mathcal{L}^{(2, \infty)}$ while $\gamma_{11}$ and $\gamma_{22}$ are Fredholm. This is a Banach-Lie group modeled on $\mathcal{L}^{(2, \infty)}\left(\mathcal{H}_{+}, \mathcal{H}_{-}\right)$. We take the space of all endomorphisms on $\mathcal{H}$ with the same condition on the off-diagonals, $\operatorname{End}_{\mathcal{L}^{(2, \infty)}}(\mathcal{H})$, and give it the natural topology under the norm $\|A\|_{+}=\left\|[\epsilon, A]_{+}\right\|+\|[\epsilon, A]\|_{\mathcal{L}^{(2, \infty)}}$. The invertible elements of $\operatorname{End}_{\mathcal{L}^{(2, \infty)}}(\mathcal{H})$, is a group, open under this topology, and it has a tangent space which comes from the natural imbedding. It is straightforward to define the "Borel subgroup";

$$
B_{1+}=\left\{\beta=\left(\begin{array}{cc}
\beta_{11} & \beta_{12} \\
0 & \beta_{22}
\end{array}\right) \mid \beta \in G L_{1+}\right\}
$$

This is the stability group of $\mathcal{H}_{-}$under the action of $G L_{1+}$ on $\mathcal{H}$. Thus the Grassmannian (which is the orbit of $\mathcal{H}_{-}$) is the complex coset space,

$$
G r_{1+}=G L_{1+} / B_{1+}
$$

It will be convenient to use the following operators for the points on $D_{1+}, \Phi: \mathcal{H} \rightarrow \mathcal{H}$,

$$
\Phi=1-2\left(\begin{array}{cc}
\left(1-Z Z^{\dagger}\right)^{-1} & -\left(1-Z Z^{\dagger}\right)^{-1} Z \\
Z^{\dagger}\left(1-Z Z^{\dagger}\right)^{-1} & -Z^{\dagger}\left(1-Z Z^{\dagger}\right)^{-1} Z
\end{array}\right)
$$

One can see that under the transformation $Z \mapsto g \circ Z, \Phi \mapsto g^{-1} \Phi g$. $\Phi$ satisfies $\epsilon \Phi^{\dagger} \epsilon=\Phi$ and $\Phi^{2}=1$. Also, $\Phi-\epsilon \in \mathcal{L}^{(2, \infty)}$, so that as an operator $\Phi$ does not differ from $\epsilon$ in an arbitrary way.

We can equivalently define the Grassmannian to be the following set of operators on $\mathcal{H}$ :

$$
G r_{1+}=\left\{\Phi \mid \Phi=\Phi^{\dagger} ; \Phi^{2}=1 ; \Phi-\epsilon \in \mathcal{L}^{(2, \infty)}\right\}
$$


Since $\Phi^{2}=1$ and it is self adjoint, it can be diagonalized by the action of

$$
U_{1+}(\mathcal{H})=\left\{g \mid g^{\dagger} g=1 ;[\epsilon, g] \in \mathcal{L}^{(2, \infty)}\right\} .
$$

Let us split $g$ into $2 \times 2$ blocks

$$
g=\left(\begin{array}{ll}
g_{11} & g_{12} \\
g_{21} & g_{22}
\end{array}\right) .
$$

The convergence condition on $[\epsilon, g]$ is the statement that the off-diagonal blocks $g_{12}$ and $g_{21}$ are in $\mathcal{L}^{(2, \infty)}$. It then follows, that $g_{11}$ and $g_{22}$ are Fredholm operators. The Fredholm index of $g_{11}$ is opposite to that of $g_{22}$; this integer is a homotopy invariant of $g$ and we can decompose $U_{1+}(\mathcal{H})$ into connected components labeled by this integer.

We can see that $U_{1+}(\mathcal{H})$ is a real form of this group. $G L_{1+}(\mathcal{H})$ is the topological product of $U_{1+}(\mathcal{H})$ and the contractible space of positive definite elements by using the fact that $\operatorname{End}_{\mathcal{L}^{(2, \infty)}}(\mathcal{H})$ has square-root of positive elements well-defined and continuous under its topology.[2]

With the projection $g \rightarrow g \epsilon g^{\dagger}$, we see that $G r_{1+}$ is a homogeneous space of $U_{1+}(\mathcal{H})$ :

$$
G r_{1+}=U_{1+}(\mathcal{H}) / U\left(\mathcal{H}_{-}\right) \times U\left(\mathcal{H}_{+}\right) .
$$

Any $\Phi \in G r_{1+}$ can be diagonalized by an element of $U_{1+}(\mathcal{H}), \Phi=g \epsilon g^{\dagger}$; this $g$ is ambiguous up to right multiplication by an element that commutes with $\epsilon$. Such elements form the subgroup

$$
U\left(\mathcal{H}_{-}\right) \times U\left(\mathcal{H}_{+}\right)=\left\{h \mid h=\left(\begin{array}{cc}
h_{11} & 0 \\
0 & h_{22}
\end{array}\right) ; h_{11}^{\dagger} h_{11}=1=h_{22}^{\dagger} h_{22}\right\} .
$$

Each point $\Phi \in G r_{1+}$ corresponds to a subspace of $\mathcal{H}$ : the eigenspace of $\Phi$ with eigenvalue -1 . Thus $G r_{1+}$ consists of all subspaces obtained from $\mathcal{H}_{-}$by an action of $U_{1+}$.

To define the tangent space at each point we can use the action of the group on itself. For our purposes it is better to take the group action on the left. Since the stability subgroup of $\epsilon$ is $U\left(\mathcal{H}_{-}\right) \times U\left(\mathcal{H}_{+}\right)$in both cases the tangent space is isomorphic to the corresponding off-diagonal algebras. In each case this is equivalent to $\mathcal{L}^{(2, \infty)}\left(\mathcal{H}_{+}, \mathcal{H}_{-}\right)$as a vector space, due to hermiticity(or psedo-hermitcity) condition.

Any given $u \in U_{1+}$ defines a vector at a given point, and a vector field can be expanded in terms of the local set of vectors. The action of a vector field on $\Phi$ is given by $V_{u(\Phi)}(\Phi)=$ $[u(\Phi), \Phi]=g\left[g^{-1} u(\Phi) g, \epsilon\right] g^{-1}$. The tangent space has a set of vectors which are given by the completion of the finite rank operators inside $\mathcal{L}^{(2, \infty)}$. This is also an ideal inside $\mathcal{B}=B(\mathcal{H})$ and is a separable Banach space under the same norm as $\mathcal{L}^{(2, \infty)}$, we will denote this set by $\left(\mathcal{L}^{(2, \infty)}\right)^{(0)}$. The tangent space has a non-canonical decomposition at each point which is isomorphic to $\left(\mathcal{L}^{(2, \infty)}\right)^{(0)}+\mathcal{L}^{(2, \infty)} /\left(\mathcal{L}^{(2, \infty)}\right)^{(0)}$, the second part is a "transversal piece". As we will see this quotient will be important for the dynamical system we have in mind.

\footnotetext{
${ }^{2}$ we can show that $U_{1+}$ is a deformation retract of $G L_{1+}$, similar to the finite dimensional case.
} 
We introduce the cotangent space as a formal expression $d \Phi$ via its contraction with the vector field at a given point; $d \Phi\left(V_{u(\Phi)}\right)=V_{u(\Phi)}(\Phi)$.

We would like to think of the $D_{1+}$ and $G r_{1+}$ as classical phase spaces. To do this we need to introduce a Poisson bracket. We will search for a symplectic form on this space. It is tempting to generalize the finite dimensional formula to this case. If we write down $\Omega=\frac{i}{4} \operatorname{Tr} \Phi d \Phi \wedge d \Phi$ we see that the trace in general does not exist. However one can see that the divergence is logarithmic, in fact the formal expression $\Phi d \Phi \wedge d \Phi$ belongs to $\mathcal{L}^{(1, \infty)}$. Hence we can replace the ordinary trace by the Dixmier trace. Dixmier trace is used in non-commutative geometry, for a masterful presentation of its properties we refer to the book and lecture notes of Connes [7].

Each choice of the trace will give another symplectic form, they all agree on the "measurable" part of the ideal $\mathcal{L}^{(1, \infty)}$. Since the "measurable" elements do not form an ideal, we cannot assume that the symplectic form is independent of the choice of the limit point.

Another important point is to remember that the Dixmier trace vanishes on the ideal generated by the completion of finite rank operators, $\left(\mathcal{L}^{(1, \infty)}\right)^{(0)}$. In the applications one expects that the operators we have to consider are pseudo-differential operators on manifolds. The physically relevant group of transformations are modeled on pseudo-differential operators which belong to the specific ideals that we have defined. In the case of classical pseudo-differential operators, the Dixmier trace is uiquely defined; it is equal to the Wodzicki residue of the pseudo-differential operator, as shown by Connes [6]. An interesting applications of Dixmier trace to the class of elliptic pseudodifferential operators is given in [15, and to chiral anomaly in [16]. An interesting discussion of the central extensions and Schwinger terms are given in [10].

In this article we will consider the general case, and show the dependence of the symplectic form to this limiting process $\omega$, explicitly on our definition of the symplectic form:

$$
\Omega_{\omega}=\frac{i}{4} \operatorname{Tr}_{\omega} \Phi d \Phi \wedge d \Phi .
$$

The existence of such a trace is the reason for our choice $\mathcal{L}^{(2, \infty)}$. One can check that the above form is closed; it is not so obvious that it is nondegenerate. In fact it vanishes whenever the result of the contractions with the vectors at a given point is in the completion of the finite rank operators inside $\mathcal{L}^{(2, \infty)},\left(\mathcal{L}^{(2, \infty)}\right)^{(0)} \neq \mathcal{L}^{(2, \infty)}$. This completion is a separable Banach space, and an ideal inside $\mathcal{B}$ as well, whereas $\mathcal{L}^{(2, \infty)}$ is a nonseperable Banach space.

The above form is invariant under the action of $U_{1+}(\mathcal{H})$ for the $G r_{1+}$ and invariant under the action of $U_{1+}\left(\mathcal{H}_{-}, \mathcal{H}_{+}\right)$for the $D_{1+}$. The formal expression is defined as

$$
i_{V_{u}} i_{V_{v}} \Omega_{\omega}=\frac{i}{8} \operatorname{Tr}_{\omega} \Phi[[u, \Phi],[v, \Phi]]
$$

\footnotetext{
${ }^{3}$ The dual space requires more care in infinite dimensions. We can think of the norm dual of $\mathcal{L}^{(2, \infty)}$, yet this space does not have a simple characterization. If we assume that the tangent space is in fact the norm dual of the cotangent space, we have a simple description of the cotangent space. We refer to Gohberg and Krein for the details [12]. In this article we will leave the question of the dual open, and use one-forms only when we have an explicit formula.
} 
One can show the invariance using this expression immediately (see below). Thus, $G r_{1+}$ and $D_{1+}$ are both homogeneous manifolds with an invariant closed two-form similar to the finite dimensional case.

Unfortunately this form is degenerate; it vanishes on the part of the tangent space which corresponds to $\left(\mathcal{L}^{(2, \infty)}\right)^{(0)}$. To see this let us calculate the contraction of $\Omega_{\omega}$ at a point $\Phi$, with a vector field which belongs to the part $\left(\mathcal{L}^{(2, \infty)}\right)^{(0)}$. Let us assume that this vector is generated by $u$ acting from the left. The condition for the vector to be in $\left(\mathcal{L}^{(2, \infty)}\right)^{(0)}$ is simply $\left[\epsilon, g^{-1} u g\right] \in\left(\mathcal{L}^{(2, \infty)}\right)^{(0)}$ at the point $\Phi=g \epsilon g^{-1}$.

$$
i_{V_{u}} \Omega_{\omega}=\frac{i}{8} \operatorname{Tr}_{\omega} \Phi[[u, \Phi], d \Phi]
$$

We will show that the contraction of this one form with an arbitrary vector on the tangent space at the same point $\Phi$ is zero, hence the form is zero. Any such vector on the tangent is again generated by the left action with a Lie algebra element $v$,

$$
i_{V_{v}} i_{V_{u}} \Omega_{\omega}=\frac{i}{8} \operatorname{Tr}_{\omega} \epsilon\left[\left[\epsilon, g^{-1} u g\right],\left[\epsilon, g^{-1} v g\right]\right]
$$

Using $\left[\epsilon,\left(\begin{array}{cc}\mathcal{B} & \mathcal{L}^{(2, \infty)} \\ \mathcal{L}^{(2, \infty)} & \mathcal{B}\end{array}\right)\right]=\left(\begin{array}{cc}0 & \mathcal{L}^{(2, \infty)} \\ \mathcal{L}^{(2, \infty)} & 0\end{array}\right)$ and similarly for the other part, we have,

$$
\left(\begin{array}{cc}
0 & \mathcal{L}^{(2, \infty)} \\
\mathcal{L}^{(2, \infty)} & 0
\end{array}\right)\left(\begin{array}{cc}
0 & \left(\mathcal{L}^{(2, \infty)}\right)^{(0)} \\
\left(\mathcal{L}^{(2, \infty)}\right)^{(0)} & 0
\end{array}\right)=\left(\begin{array}{cc}
\left(\mathcal{L}^{(1, \infty)}\right)^{(0)} & 0 \\
0 & \left(\mathcal{L}^{(1, \infty)}\right)^{(0)}
\end{array}\right)
$$

where we use $\left(\mathcal{L}^{(2, \infty)}\right)^{(0)} \mathcal{L}^{(2, \infty)} \in\left(\mathcal{L}^{(1, \infty)}\right)^{(0)}$ (see Appendix for a proof). The Dixmier trace vanishes on $\left(\mathcal{L}^{(1, \infty)}\right)^{(0)}$ and this shows that the form is zero. In general since the tangent space has the direction given by $\left(\mathcal{L}^{(2, \infty)}\right)^{(0)}$, the cotangent space has one-forms which do not vanish on them. If $\Lambda$ is a form such that $\Lambda\left(V_{u}\right) \neq 0$, then $\Lambda=i_{Y} \Omega_{\omega}$ has no solution for the vector $Y$. If we assume that the de Rham theory makes sense on these spaces, since $D_{1+}$ is contractible, and $\pi_{1}\left(G r_{1+}\right)=0$, we would expect that there is a function $f$ such that $\Lambda=d f$, and this will show that one cannot obtain Hamiltonian vector field for any given function $f$ in general. Nevertheless, as we will see, for the relevant part of the space, that is for "large" motions, directions which belong to $\mathcal{L}^{(2, \infty)} /\left(\mathcal{L}^{(2, \infty)}\right)^{(0)}$, the form is non-degenerate. This will allow us to define classical dynamics for certain systems.

Before we continue, let us point out an important observation. For clarity let us concentrate on $G r_{1+}$. There is an interesting leaf of $G r_{1+}$ which corresponds to the orbit of $\epsilon$ under the following subgroup;

$$
U_{1+}^{(0)}=\left(\begin{array}{cc}
\mathcal{B} & \left(\mathcal{L}^{(2, \infty)}\right)^{(0)} \\
\left(\mathcal{L}^{(2, \infty)}\right)^{(0)} & \mathcal{B}
\end{array}\right)
$$

\footnotetext{
${ }^{4}$ This means that we throw away a large part of the symplectic manifold. Perhaps a physically more appropriate choice is to use a combination, which keeps the information about the "small" directions. Although this can be done we will focus on the above symplectic form.
} 
We denote this orbit by $G r_{1+}^{(0)}$. Since the connected components are still labeled by the integers, each connected component of $G r_{1+}$ has the connected component of $G r_{1+}^{(0)}$ inside. If we take $G r_{1+}^{(0)}$ 's own tangent space, generated by $U_{1+}^{(0)}$, the symplectic form vanishes on this orbit using the fact that $\left(\mathcal{L}^{(2, \infty)}\right)^{(0)} \mathcal{L}^{(2, \infty)} \in\left(\mathcal{L}^{(1, \infty)}\right)^{(0)}$,(see Appendix for a proof). The same remarks apply to the Disc $D_{1+}$, and we spare the details for the reader.

Since the group action preserves the two from $\Omega_{\omega}$ the Lie derivative along the direction of any vector field generated by the group action gives us zero. This raises the possibility of finding the moment maps which would generate the infinitesimal action of $U_{1+}\left(\mathcal{H}_{-}, \mathcal{H}_{+}\right)$and $U_{1+}(\mathcal{H})$ respectively. We can start with the finite dimensional answer; one can check that to avoid the divergence the finite dimensional answer has to be modified as $-\operatorname{Tr}_{\omega}^{\epsilon} u(\Phi-\epsilon)$, where $u$ is a hermitian matrix which is in the Lie algebra of $U_{+}(\mathcal{H})$ for the $G r_{1+}$ and a pseudo hermitian $\left(u^{\dagger}=\epsilon u \epsilon\right)$ operator which belongs to $U_{1+}\left(\mathcal{H}_{-}, \mathcal{H}_{-}\right)$for $D_{1+}$. We use a conditionally convergent trace for the variable $\Phi-\epsilon$ if we think of the group acting from the left. To see that the above expression makes sense consider $u \in\left(\begin{array}{cc}\mathcal{B} & \mathcal{L}^{(2, \infty)} \\ \mathcal{L}^{(2, \infty)} & \mathcal{B}\end{array}\right)$, and $\Phi-\epsilon \in\left(\begin{array}{ll}\mathcal{L}^{(1, \infty)} & \mathcal{L}^{(2, \infty)} \\ \mathcal{L}^{(2, \infty)} & \mathcal{L}^{(1, \infty)}\end{array}\right)$. (Here $\mathcal{B}$ is the space of bounded operators.) As a result $u(\Phi-\epsilon) \in\left(\begin{array}{ll}\mathcal{L}^{(1, \infty)} & \mathcal{L}^{(2, \infty)} \\ \mathcal{L}^{(2, \infty)} & \mathcal{L}^{(1, \infty)}\end{array}\right)$. Let us define $M=\Phi-\epsilon$. Formally the equation for the group action is satisfied,

$$
-\operatorname{Tr}_{\omega}^{\epsilon} u d \Phi=\Omega_{\omega}\left(V_{u}, \quad\right) \quad V_{u}(\Phi)=[u, \Phi]
$$

but we must be careful since if $g^{-1} u g \in\left(\mathcal{L}^{(2, \infty)}\right)^{(0)}$ the right handside vanishes as we have shown. The only way to satisfy this equation, is to show that whenever the right handside vanishes the left vanishes as well. This can be checked as follows;

$$
d f_{u}\left(V_{v}\right)=\operatorname{Tr}_{\omega}^{\epsilon} u[v, \Phi]=\operatorname{Tr}_{\omega}^{\epsilon} g^{-1} u g\left[\epsilon, g^{-1} v g\right]
$$

for any $v \in U_{1+}$. By the same argument as above, we see that the resulting expression inside the trace is of class $\left(\mathcal{L}^{(1, \infty)}\right)^{(0)}$. Hence the trace is also zero.

We notice that the form corresponding to the moment map $-\operatorname{Tr}_{\omega}^{\epsilon}\left(u M^{(0)}\right)$ for any $u \in U_{1+}$ when contracted with the elements of the tangent space of $D_{1+}^{(0)}$ or $G r_{1+}^{(0)}$ also gives zero. This is in some sense the orbit one can neglect; we can think of it as the null orbit. This shows that we cannot take the separable part in our definition of the group $U_{1+}$, denoted as $\left(U_{1+}\right)^{(0)}$, if we use the Dixmier trace. In a recent preprint [8], it is argued that there is no positive trace on the ideal $\left(\mathcal{L}^{(2, \infty)}\right)^{(0)}$, this implies that a similar construction cannot be achived for the group $\left(U_{1+}\right)^{(0)}$.

However, it is not enough to show that the moment functions satisfy a consistent equation. Normally in the Hamiltonian formalism we are given a function and asked to find the vector field generated by this function. Our discussion shows that this vector field at every point is only determined in the eqivalence class $\mathcal{L}^{(2, \infty)} /\left(\mathcal{L}^{(2, \infty)}\right)^{(0)}$. It is easy to see that if we take instead of $u$, a vector generated by $u+v$ such that $g^{-1} v g \in\left(\mathcal{L}^{(2, \infty)}\right)^{(0)},-d f_{u}=\frac{i}{8} \operatorname{Tr}_{\omega} \Phi[[u+$ $v, \Phi], d \Phi]=\frac{i}{8} \operatorname{Tr}_{\omega} \Phi[[u, \Phi], d \Phi]+\frac{i}{8} \operatorname{Tr}_{\omega}[[v, \Phi], d \Phi]$ for the last piece is zero by the previous 
argument. This implies that the moment funtions do not have unique vector fields, they generate the motions in the "transversal direction" $\mathcal{L}^{(2, \infty)} /\left(\mathcal{L}^{(2, \infty)}\right)^{(0)}$ with an undetermined piece in $\left(\mathcal{L}^{(2, \infty)}\right)^{(0)}$. Let us look at the infinitesimal part of this evolution for two different choices of the vector field in the equivalence class; $\Delta \Phi=t[u, \Phi]$ and $\Delta^{\prime} \Phi=t\left[u^{\prime}, \Phi\right]$. The difference of these two infinitesimal evolutions are given by $\Delta \Phi-\Delta^{\prime} \Phi=\left[u-u^{\prime}, \Phi\right]=g\left[g^{-1}(u-\right.$ $\left.\left.u^{\prime}\right) g, \epsilon\right] g^{-1}$. Since the ambiguity is a result of the difference, which satisfies $\left[g^{-1}\left(u-u^{\prime}\right) g, \epsilon\right] \in$ $\left(\mathcal{L}^{(2, \infty)}\right)^{(0)}$; this term is in the orbit $D_{1+}^{(0)}$, or in the same connected component of $G r_{1+}^{(0)}$. Thus the difference of the infinitesimal evolutions can be conjugated to the orbit $D_{1+}^{(0)}$, or to the same connected component of $G r_{1+}^{(0)}$. This implies that the relevant space for the classical dynamics is not the original quotient we look, but a smaller one, given by $U_{1+} / U_{1+}^{(0)}$. In fact as we will see later on, this reduction has an interesting consequence. But, for the moment we will continue to use the "unreduced phase space".

To talk about classical evolution, we will make the proposal that this type of classical systems are defined through an equivalence relation. We will assume that two dynamical evolutions are equivalent if they could be conjugated to the "null" orbit, $D_{1+}^{(0)}$, for $D_{1+}$ and to the same connected component of $G r_{1+}^{(0)}$, for $G r_{1+}$. Another way to think about this is that the dynamics in the "small" directions cannot be determined. The moment functions we consider only determine the evolution under equivalence.

This feature will affect the dynamics generated by Hamiltonians of more complicated functions. The generic Hamiltonians we have in mind are quadratic functions of the variable $M=\Phi-\epsilon$ plus a moment map. We think of the moment map as the free part of the Hamiltonian since it generates the goup action upto an equivalence, and the quadratic piece as an "interaction".

Formally we can write the Hamiltonians as,

$$
h=i \operatorname{Tr}_{\omega}^{\epsilon} u M+\operatorname{Tr}_{\omega}^{\epsilon} \hat{K}(M) M
$$

where, $\hat{K}$ is a linear operator which acts on the variable $M$. If we specify a basis, it can be expressed as $(\hat{K}(M) M)_{p}^{k}=\sum_{i j k} K_{j l}^{i k} M_{i}^{j} M_{p}^{l}$. The summability properties of the kernel $K_{j l}^{i k}$ should be such that the resulting operator is in the ideal $\mathcal{L}^{(1, \infty)}$. For example, this can be achieved, if the map $\hat{K}$ produces an operator in the group $U_{1+}$. Notice that it is important to use the Dixmier trace again. As we mentioned in the moment maps, since the symplectic form $\Omega_{\omega}$ vanishes upon contraction with elements of the tangent space in the "small" directions, the interaction hamiltonian must have the same property. As one can check this form of the Hamiltonian, when differentiated, gives a form which vanishes on the same subspace. This will impose certain conditions on the choice of kernels. Interactions which are "too weak" will not affect the equations of motion.

Let us give a typical interaction Hamiltonian. We can take two moment functions and take their products;

$$
\sum_{i j} K^{i j} \operatorname{Tr}_{\omega}^{\epsilon} u_{i} M \operatorname{Tr}_{\omega}^{\epsilon} u_{j} M
$$


If the sum is over a finite number of terms then clearly this is a well-defined expression. In general one should be able to choose $K^{i j}$ such that the above expression is finite. For these Hamiltonians, one can directly calculate the equations of motion by using the Poisson bracket relations among the moment functions only. As we will see later on, they also have a simpler description in the quantum case.

A word of caution should be said here. Typically, the Hamiltonians are more singular than the symplectic form, and they require further renormalizations. This implies a choice of domain for the Hamiltonian. This should restrict the accessible regions of the phase space, or the true phase space of the theory, and it may change the formulation of the problem drastically. In real physical systems, we expect these problems to modify the precise formulation of the field theory. In this work, we do not discuss this more difficult problem. In some sense this is part of the kinematics, although interactions may even change this.

There will always be vector fields generating the equations of motion upto equivalence. In infinite dimensions determination of the integral curves and their completeness are highly non-trivial issues; the answer depends on the Hamiltonian as well. This is the classical version of the unitarity condition in quantum mechanics.

From general principles we expect that the Poisson brackets of the moment functions will provide a realization of the corresponding Lie algebra possibly with a central extension. We can calculate the Poisson bracket of two moment functions using a formal manipulation and it gives us,

$$
\left\{f_{u}, f_{v}\right\}=f_{-i[u, v]}-i \operatorname{Tr}_{\omega}^{\epsilon}[\epsilon, u] v .
$$

This is a central extension of the full group, indexed by the choice of limit process $\omega$. (Note that there is no ambiguity in this relation since the right handside is the same for equivalent choices of the vector fields). One can explicitly check that $c_{\omega}(u, v)=\operatorname{Tr}_{\omega}^{\epsilon}[\epsilon, u] v$ satisfies $c_{\omega}(u, v)=-c_{\omega}(v, u)$ and the cocycle condition, $c_{\omega}(u,[v, w])+c_{\omega}(v,[w, u])+c_{\omega}(w,[u, v])=0$. Since the central term vanishes on the ideal $\left(\mathcal{L}^{(1, \infty)}\right)^{(0)}$ it will not be there when $u$ or $v \in U_{1+}^{(0)}$. The actual computation should be done with care, due to infinite dimensionality. One can see that the both sides of the equation after explicit calculation give the same result, hence they are identical. We will leave the details to the reader.

This gives us a symplectic realization of the Lie algebra of $U_{1+}(\mathcal{H})$ for $G r_{1+}$ and $U_{1+}\left(\mathcal{H}_{-}, \mathcal{H}_{+}\right)$ for $D_{1+}$ except a central term. Since the calculation of the both sides are actually zero whenever $g^{-1} u g$ or $g^{-1} v g \in U_{1+}^{(0)}$, this expression should be thought of as a realization of the "large" part of the Lie algebra. Đ Let us consider this central extension for the pseudodifferential operators $\left[\right.$; then we have $\operatorname{Tr}_{\omega}[\epsilon, u] v=\operatorname{Res}([\epsilon, u] v)$. We recall that the residue is actually defined for all pseudodifferential operators and it satisfies $\operatorname{Res}[A, B]=0[23$, 11]. This implies that, in fact $\operatorname{Tr}_{\omega}[\epsilon, u] v=\partial \phi(u, v)$, for $\phi(u)=\operatorname{Res}(\epsilon u)$, hence the central extension is trivial.

Because of the infinite dimensionality an attempt to remove the central term, in the

\footnotetext{
${ }^{5}$ It is more natural to look into the spaces which are modeled on the quotients $\mathcal{L}^{(2, \infty)} /\left(\mathcal{L}^{(2, \infty)}\right)^{(0)}$. Since $\left(\mathcal{L}^{(2, \infty)}\right)^{(0)}$ is closed the quotient is well-defined. Later on we will consider this point of view.

${ }^{6}$ This is pointed out to me by Mickelsson
} 
genral case, will result in a divergent expression. This gives us the Lie algebra of the nontrivial central extension of $U_{1+}$ corresponding to $\omega$. We expect that these extensions are not equivalent in general for different choices of $\omega$ but they all agree on the subset modelled on the "measurable" elements-this is not a subalgebra of $\underline{U_{1+}}$, so one cannot reduce it to this case.

\section{Quantization}

We continue to think of classical mechanics in geometric terms [2, [1]. Let us assume that the phase space, $\Gamma$ is a smooth manifold. If we have a Poisson structure on the algebra of smooth functions $C^{\infty}(\Gamma)$, we can introduce classical dynamics. Quantization of this classical system is given by a representation of the Poisson algebra of smooth functions by self-adjoint operators on a Hilbert space. This is an overambitious program; in general there is no way to find such a representation. The difficulties and various methods have been well-explained in the literature 3, 13, 14]. In this article we will follow our point of view in [21]. We will find a represention of the Poisson algebra of moment functions. Any composite function, which is related to the product of two moment maps can be qantized by giving an ordering rule. We will not attempt to establish this idea in the present article.

Before we proceed further, we need to make a digression and introduce a generalized "determinant". It does not satisfy all the properties of a determinant. As we will see, to think of it as a determinant simplifies the calculations. Let us $\operatorname{define} \operatorname{det}_{\omega}(1+A)$ for $A \in \mathcal{L}^{(1, \infty)}$ as $\mathrm{Q}$

$$
\operatorname{det}_{\omega}(1+A)=\exp \left(\operatorname{Tr}_{\omega}(A)\right)
$$

One can see that it satisfies the multiplicative property of the determinants;

$$
\operatorname{det}_{\omega}((1+A)(1+B))=\operatorname{det}_{\omega}(1+A) \operatorname{det}_{\omega}(1+B),
$$

due to $\mathcal{L}^{(1, \infty)} \mathcal{L}^{(1, \infty)} \in \mathcal{L}^{1}$ and $\operatorname{Tr}_{\omega}$ vanishes on the trace class operators $\mathcal{L}^{1}$. An interesting property is that $\operatorname{det}_{\omega}$ never vanishes.

We will use this to provide a representation of the borel subroup on $\mathbf{C}$, and attempt to follow the geometric quantization program.

We will introduce an ad hoc representation, which comes from the geometric quantization performed in [21], of the Lie algebra of the group $U_{1+}\left(\mathcal{H}_{-}, \mathcal{H}_{+}\right)$on the space of "holomorphic

\footnotetext{
${ }^{7}$ One way to motivate this definition is the following. Let us take the determiant formula, $\log \operatorname{det}(1+A)=$ $\operatorname{Tr} \log (1+A)$. We replace the trace by the Dixmier trace, and $\operatorname{define} \log \operatorname{det}_{\omega}(1+A)=: \operatorname{Tr}_{\omega} \log (1+A)=$ $\operatorname{Tr}_{\omega}\left(A-\frac{1}{2} A^{2}+\frac{1}{3} A^{3}-\ldots\right)=\operatorname{Tr}_{\omega}(A)$ since all the higher terms are in $\mathcal{L}^{1}$ and the Dixmier trace vanishes on them. Ths gives the above formula again.
} 
functions"

$$
\hat{f}_{-u} \Psi(Z)=-i \hbar\left[\mathcal{L}_{V_{u}} \Psi(Z)-\frac{1}{\hbar} \operatorname{Tr}_{\omega}(\gamma Z) \Psi(Z)\right],
$$

where $u \in \underline{U}_{1}\left(\mathcal{H}_{-}, \mathcal{H}_{+}\right)$, and $V_{u}=V_{u}^{Z} \partial_{Z}+V_{u}^{Z^{\dagger}} \partial_{Z^{\dagger}}$ is the formal vector field generated by the action of $-u$. It is easier to define the Lie derivative directly, by using the action of the Lie algebra on the Disc. We define this as,

$$
\mathcal{L}_{V_{u}} \Psi(Z)=\lim _{t \rightarrow 0} \frac{\Psi(Z+t(\alpha Z+\beta-Z \gamma Z-Z \delta))-\Psi(Z)}{t},
$$

where,

$$
u=\left(\begin{array}{ll}
\alpha & \beta \\
\gamma & \delta
\end{array}\right)
$$

is the decomposition of $u$ into block form; $\alpha^{\dagger}=-\alpha, \beta^{\dagger}=\gamma$ and $\delta^{\dagger}=-\delta$ and further $\gamma, \beta \in \mathcal{L}^{(2, \infty)}$. We notice that this differs from the finite dimensional answer by a constant term, which is infinite in this case. The changes introduced in the functions are all "holomorphic", so the action of the operators corresponding to the moment functions preserve the "holomorphicity condition". In order to show that this is the correct representation, we need to prove that the commutation relations are satisfied acting on these set of functions.

let us check that the commutation relations will give us a realization of the Poisson bracket relations satisfied by the moment maps.

$$
\left[\hat{f}_{-u_{1}}, \hat{f}_{-u_{2}}\right] \Psi(Z)=i \hbar \hat{f}_{\left[u_{1}, u_{2}\right]} \Psi(Z)+i \hbar \operatorname{Tr}_{\omega}^{\epsilon}\left(\left[\epsilon, u_{1}\right] u_{2}\right) \Psi(Z) .
$$

A calculation shows that,

$$
\left.\left[\hat{f}_{-u_{1}}, \hat{f}_{-u_{2}}\right] \Psi(Z)=\hbar^{2}\left(\mathcal{L}_{u_{1}} \mathcal{L}_{u_{2}}-\mathcal{L}_{u_{2}} \mathcal{L}_{u_{1}}\right) \Psi(Z)+\hbar\left[\left(\mathcal{L}_{u_{1}} \operatorname{Tr}_{\omega} \gamma_{2} Z\right)-\left(\mathcal{L}_{u_{2}} \operatorname{Tr}_{\omega} \gamma_{1} Z\right)\right)\right] \Psi(Z) .
$$

For simplicity we use $\mathcal{L}_{u}$ instead of $\mathcal{L}_{V_{u}}$. This is equal to

$$
\left[\hat{f}_{-u_{1}}, \hat{f}_{\left.-u_{2}\right]}\right] \Psi(Z)=\hbar^{2} \mathcal{L}_{\left[u_{1}, u_{2}\right]} \Psi(Z)+\hbar \operatorname{Tr}_{\omega}\left\{\gamma\left(\left[u_{1}, u_{2}\right]\right) Z\right\} \Psi(Z)+\hbar \operatorname{Tr}_{\omega}\left(\gamma_{1} \beta_{2}-\gamma_{2} \beta_{1}\right) \Psi(Z)
$$

The last term, which is a constant multiple, can be rewritten as $\operatorname{Tr}_{\omega}^{\epsilon}\left[\epsilon, u_{1}\right] u_{2}$. Hence we see that it is a representation of the Poisson bracket relations (24).

This representation can in fact be integrated to a representation of the group action on the space of holomorphic functions:

$$
\rho_{\omega}\left(g^{-1}\right) \Psi=\operatorname{det}_{\omega}^{-\frac{1}{\hbar}}\left(d^{-1} c Z+1\right) \Psi\left((a Z+b)(c Z+d)^{-1}\right) .
$$

\footnotetext{
${ }^{8}$ The author is not aware of a well-established definition of holomorphicity for infinite dimensions. We assume that the algebraic operations on the coordinate $Z$ after the application of a dual element, if it is finite, provides a holomorphic function. This will be used for the grassmanian as well.
} 
These representations are labelled by $\omega$, the choice of limit point, and $\hbar$. Since the determinant never vanishes and actually given by an exponential $\hbar$ is any real number. I To justify this, we will compute the infinitesimal form of the representation and show that it is given by the operators corresponding to the moment maps. We write explicitly,

$$
\rho\left(g^{-1}\right) \Psi(Z)=\mathrm{e}^{-\frac{1}{\hbar} \operatorname{Tr}_{\omega}\left(d^{-1} c Z\right)} \Psi(g \circ Z)
$$

and evaluate,

$$
\lim _{t \rightarrow 0}\left\{\frac{\rho_{\omega}(1+t u)-\rho_{\omega}(1)}{t}\right\} \Psi(Z)=\hbar\left[\mathcal{L}_{V_{u}}-\frac{1}{\hbar} \operatorname{Tr}_{\omega}(\gamma Z)\right] \Psi(Z) .
$$

So we see that the infinitesimal form is given by the moment map operators. We still have to check that this is a representation;

$$
\rho_{\omega}\left(g_{1}\right) \rho_{\omega}\left(g_{2}\right) \Psi(Z)=c_{\omega}\left(g_{1}, g_{2}\right) \rho_{\omega}\left(g_{1} g_{2}\right) \Psi(Z)
$$

where $c_{\omega}\left(g_{1}, g_{2}\right)$ is a central term, which satisfies, $c_{\omega}\left(g_{1} g_{2}, g_{3}\right) c_{\omega}\left(g_{1}, g_{2}\right)=c_{\omega}\left(g_{1}, g_{2} g_{3}\right) c_{\omega}\left(g_{2}, g_{3}\right)$. Since the Disc is topologically trivial the central extension can be described by specifying a function from the cartesian product of the group to C. An explicit calculation which is given in the Appendix, shows that the group property is satisfied with a central term,

$$
c_{\omega}\left(g_{1}, g_{2}\right)=\operatorname{det}_{\omega}^{\frac{1}{\hbar}}\left[\left(d_{1} d_{2}\right)^{-1} c_{1} b_{2}+1\right]=\exp \left(\frac{1}{\hbar} \operatorname{Tr}_{\omega}\left[\left(d_{1} d_{2}\right)^{-1} c_{1} b_{2}\right]\right) .
$$

But there is still one more point we need to consider. Recall that the orbit of $\epsilon$ corresponding to the subgroup $U_{1+}^{(0)}, D_{1+}^{(0)}$, when considered as a submanifold with its own tangent space has no dynamics under our choice of the symplectic form. Hence the Poisson algebra of moment maps restricted on this submanifold is trivially true, being zero on both sides. Whereas the representation space we choose, when restricted to the subspace $D_{1+}^{(0)}$ provide a nontrivial representation of the Poisson algebra of moment maps. This can be rectified by selecting a subspace of holomorphic functions which remain constant on the orbit $D_{1+}^{(0)}$. As a result, we define the Quantum Hilbert space to be

$$
\mathcal{H}_{Q}=\left\{\Psi(Z) \mid \Psi(Z) \text { holomorphic on } D_{1+} \text { and }\left.\Psi\right|_{D_{1+}^{(0)}}=\text { constant }\right\}
$$

This condition is consistent with the assumption that the dynamics along the directions $\left(\mathcal{L}^{(2, \infty)}\right)^{(0)}$ is unimportant. If we consider $\epsilon$ to be the "vacuum" configuration, its orbit under $U_{1+}^{(0)}$ is equivalent to this "vacuum" configuration.

For this choice of the Quantum Hilbert space, we will exhibit a class of wave functions. We are unable to prove that these are the only possible ones. Since the wave functions should be constant on the orbit $D_{1+}^{(0)}$, it suggests the use of the Dixmier trace again.

\footnotetext{
${ }^{9}$ This point of view on quantization for finite dimensional homogeneous spaces appeared in [5]
} 
We can compose polynomials in the variable $Z$. The interesting thing is to note that we can go upto quadratic terms only inside the Dixmier trace. For any choice of $A_{i} \in \mathcal{L}^{(2, \infty)}$, and any two $B_{j}, B_{k} \in B(\mathcal{H})$, we can form,

$$
\operatorname{Tr}_{\omega}\left(A_{i} Z\right) \& \operatorname{Tr}_{\omega}\left(B_{j} Z B_{k} Z\right)
$$

Using the generalized Hölder inequality [22], we have the inequalities,

$$
\left|\operatorname{Tr}_{\omega}\left(A_{i} Z\right)\right| \leq\left\|\left.A_{i}\right|_{\mathcal{L}^{(2, \infty)}}|| Z\right\|_{\mathcal{L}^{(2, \infty)}} \quad \text { and } \quad\left|\operatorname{Tr}_{\omega}\left(B_{k} Z B_{j} Z\right)\right| \leq\left\|B_{k}|\||\left|B_{j}\right||||Z|_{\mathcal{L}^{(2, \infty)}}^{2}\right.
$$

These show the continuity in $Z$ and with respect to $A_{i}$ and $B_{j}, B_{k}$. We can compose products of these kind of functions;

$$
\Psi(Z)=\prod_{i, j, k} \operatorname{Tr}_{\omega}\left(B_{j} Z B_{k} Z\right) \operatorname{Tr}_{\omega}\left(A_{i} Z\right)
$$

The reader can verify that any higher power of $Z$ is irrelevant, so these are the only combinations we can make. Various superpositions of these functions will give us the set of wave functions. I0

We also would like to point out an interesting property of our wave functions. Since we have obtained them through the Dixmier trace, we effectively perform "logarithmic wave function renormalization".

The Quantum Hilbert space may be very large, or maybe very small, it depends on the size of the space of "holomorphic functions" on $D_{1+}$, and the inner product. It is not clear how one should introduce a measure to define an inner product in this Quantum Hilbert space. The term Hilbert space is only justified by thinking of this as a quantization of a classical system. It should be possible to extend the work in [19] to this case. Once this is done, the completion of the above set of wave functions with respect to this measure, will be the quantum Hilbert space, $\mathcal{H}_{Q}$.

Next we will construct the quantum operators for the $G r_{1+}$. Our approach will be somewhat ad hoc again. Since $G r_{1+}$ is topologically nontrivial, it may not be possible to represent wave functions as functions on the grassmannian. It is natural to introduce them as sections of a complex line bundle. However, we will see that there are non-constant holomorphic functions on this grassmanian."1 For the quantization of our classical system,

\footnotetext{
${ }^{10}$ One should perhaps compare this with the analysis given in [21] There, the holomorphic wave functions can be constructed using the analogy with the finite dimensional case. We can use the fact that the dual of $\mathcal{L}^{2}$ is itself and the dual of $\mathcal{L}^{1}$ is $B(\mathcal{H})$. We can write down a general holomorphic function as sums and products of the expressions of the form, $\operatorname{Tr}(A Z), \operatorname{Tr}\left(B_{1} Z B_{2} Z \ldots B_{m} Z\right)$, where $A \in \mathcal{L}^{2}$ and $B_{k} \in B(\mathcal{H})$ for all $k=1, . . m$. Not all of them are linearly independent of course. With the appropriate inner product the Quantum Hilbert space constructed out of these functions is isomorphic to the usual Fock space and it is a seperable Hilbert space.

${ }^{11}$ This can be contrasted with the [18, 21], there, the finite dimensional grassmanians are dense inside the full grassmanian, and it is well-known that on a compact complex manifold there are no non-constant holomorphic functions. In our case, completion of finite rank objects will not be equal to the full space.
} 
we actually need the sections of a line bundle. For this it is better to think of $G r_{1+}$ as a qoutient of another pair of groups as in the case discussed by [18, 21]. It is possible that the extension is nontrivial both topologically and algebraically. Essentially, we will enlarge 'numerator' and 'denominator' by the same amount, this will keep the ratio same. We define the group $\tilde{G}_{1+}$ :

$$
\tilde{G}_{1+}=\left\{(\gamma, q) \mid q \in G L\left(\mathcal{H}_{-}\right) ; \quad \gamma \in G L_{1+}(\mathcal{H}), \quad \gamma_{11} q^{-1}-1 \in \mathcal{L}^{(1, \infty)}\right\} .
$$

Here, $\gamma_{11}$ denotes the mapping $\gamma_{11}: \mathcal{H}_{-} \rightarrow \mathcal{H}_{-}$in the block form of the matrix $\gamma \in G L_{1+}(\mathcal{H})$. One can prove that the set of $q$ 's which satisfy this condition is not empty using the definition of the group $G L_{1+}$ following Pressley and Segal [18]. We can give a topology to this space using the two topologies inherited from the bounded and $\mathcal{L}^{(1, \infty)}$. (Notice that the extension for any two sided ideal is mentioned in [18 at page 98.)

$\tilde{G}_{1+}$ is a complex Banach-Lie group under the multiplication $(\gamma, q)\left(\gamma^{\prime}, q^{\prime}\right)=\left(\gamma \gamma^{\prime}, q q^{\prime}\right)$. We introduce $\tilde{B}_{1+}$, a closed complex subgroup of $\tilde{G}_{1+}$;

$$
\tilde{B}_{1+}=\left\{(\beta, t) \mid \beta \in B_{1+}, t \in G L\left(\mathcal{H}_{-}\right), \beta_{11} t^{-1}-1 \in \mathcal{L}^{(1, \infty)}\right\}
$$

There is an action of $\tilde{B}_{1+}$ on $\tilde{G}_{1+}$. Since this action does not involve anything but multiplication in the group, it is holomorphic. We enlarged $G L_{1+}(\mathcal{H})$ and $B_{1+}$ with the same set of elements, thus the quotient is still the same;

$$
\tilde{B}_{1+} \rightarrow \tilde{G}_{1+} \rightarrow G r_{1+}
$$

In this case as well, there are subgroups corresponding to completions of the finite rank elements. These subgroups now can be written as,

$$
\tilde{G}_{1+}^{(0)}=\left\{\left(\gamma^{(0)}, q\right) \mid q \in G L\left(\mathcal{H}_{-}\right) ; \quad \gamma^{(0)} \in G L_{1+}^{(0)}(\mathcal{H}), \quad \gamma_{11}^{(0)} q^{-1}-1 \in\left(\mathcal{L}^{(1, \infty)}\right)^{(0)}\right\}
$$

Existence can be proved along the same lines. The stability subgroup also changes to $\tilde{B}_{1+}^{(0)}$, which is defined similarly. One can see that $\tilde{G}_{1+}^{(0)} \subset \tilde{G}_{1+}$ as a closed subset. This subgroup will correspond to the null orbit, and it will not have any extension.

Now, we can introduce the holomorphic line bundle corresponding to the representation $\rho(\beta, r)=\operatorname{det}_{\omega}^{\frac{1}{\hbar}}\left(\beta_{11} r^{-1}\right)$. There is no condition for the number $\hbar$ to be an integer at this stage, since our definition for $\operatorname{det}_{\omega}$ has an exponential, it never vanishes and the value of $\hbar$ could be any real number. We denote the line bundle as $\left(\tilde{G}_{1+} \times_{\rho} \mathbf{C}\right) / \tilde{B}_{1+}$. A section of this line bundle can be identified with equivariant functions:

$$
\psi: \tilde{G}_{1+} \rightarrow \mathbf{C} \quad \text { such that } \psi(\gamma \beta, q r)=\rho(\beta, r) \psi(\gamma, q) .
$$

Let us exhibit the functions which would satisfy this condition. They are given by generalized determinants very similar to the case discussed in [21], as an example we start with,

$$
\psi(\gamma, q)=\operatorname{det}^{\frac{1}{\hbar}}\left(\gamma_{11} q^{-1}\right)
$$


One can see that this is an equivariant function on the space $\tilde{G}_{1+}$, using the properties of the Dixmier trace. There is no restriction on the value of $\hbar$ due to holomorphicity, since the exponential is an entire function except possibly due to a topological obstruction. We can compose more functions of this type, if we allow "mixing" of the elements of $\gamma_{11}$ with the elements of $\gamma_{21}$, in a controlled way. One can see that if we assume that the mixing is allowed by finite rank operators the result will not change. More than that, it will not change if we use elements of $\left(\mathcal{L}^{(2, \infty)}\right)^{(0)}$. To get different functions we need to mix them by elements of $\mathcal{L}^{(2, \infty)} /\left(\mathcal{L}^{(2, \infty)}\right)^{(0)}$.

$$
\operatorname{det}_{\omega}\left(\left(1-A_{i} S\right) \gamma_{11} q^{-1}+A_{i} \gamma_{21} q^{-1}\right)
$$

for $A_{i} \in \mathcal{L}^{(2, \infty)}\left(\mathcal{H}_{+}, \mathcal{H}_{-}\right)$and the mapping $S: \mathcal{H}_{-} \rightarrow \mathcal{H}_{+}$is an isometric isomorphism which is given by mapping one set of orthonormal basis elements into the other( it can simply be taken as sending $e_{-i} \rightarrow e_{i}$ ). This form is guessed from the system studied in [21, [18]. However we have two problems with this form. When we use $A_{i} S \gamma_{11}$, this expression is not convergent under Dixmier trace. The second is that we want our wave functions to be constant when they are restricted to the null orbit, as we will see this is not possible for the above form of the functions due to the term $A_{i} S \gamma_{11}$. One can see that dropping this term does not change the equivariance condition thanks to the Dixmier trace again. This means an infinite multiplicative renormalization. At the same time we see that it is simpler to just multiply with the function $\operatorname{Tr}_{\omega}\left(A_{i} \gamma_{21} q^{-1}\right)$, since it is invariant under the action of $\tilde{B}_{1+}$, hence it descends to a function on the quotient, $G r_{1+}$. A similar argument shows that we can do better, we may add even a nonlinear term, which is still invariant. Any higher order addition vanishes. We can write down a general expression,

$$
\operatorname{det}_{\omega}^{\frac{1}{\hbar}}\left(\gamma_{11} q^{-1}\right) \operatorname{Tr}_{\omega}\left(A_{i} \gamma_{21} q^{-1}\right) \operatorname{Tr}_{\omega}\left(B_{j} \gamma_{21} q^{-1} B_{k} \gamma_{21} q^{-1}\right)
$$

where $B_{j}, B_{k} \in B\left(\mathcal{H}_{+}, \mathcal{H}_{-}\right)$. For clarity, we will prove that this form is equivariant and satisfies all the requirements.

Let us look at the action by an element of $\tilde{B}_{1+}$;

$$
\begin{aligned}
& \operatorname{det}_{\omega}^{\frac{1}{\hbar}}\left((\gamma \beta)_{11}(q r)^{-1}\right) \operatorname{Tr}_{\omega}\left(A_{i}(\gamma \beta)_{21}(q r)^{-1}\right) \operatorname{Tr}_{\omega}\left(B_{j}(\gamma \beta)_{21}(q r)^{-1} B_{k}(\gamma \beta)_{21}(q r)^{-1}\right)= \\
& =\operatorname{det}_{\omega}^{\frac{1}{\hbar}}\left(\gamma_{11} \beta_{11} r^{-1} q^{-1}\right) \operatorname{Tr}_{\omega}\left(A_{i} \gamma_{21} \beta_{11} r^{-1} q^{-1}\right) \operatorname{Tr}_{\omega}\left(B_{j} \gamma_{21} \beta_{11} r^{-1} q^{-1} B_{k} \gamma_{21} \beta_{11} r^{-1} q^{-1}\right)= \\
& =\operatorname{det}_{\omega}^{\frac{1}{\hbar}}\left(\gamma_{11}(1+I) q^{-1}\right) \operatorname{Tr}_{\omega}\left(A_{i} \gamma_{21}(1+I) q^{-1}\right) \operatorname{Tr}_{\omega}\left(B_{j} \gamma_{21}(1+I) q^{-1} B_{k} \gamma_{21}(1+I) q^{-1}\right)
\end{aligned}
$$

using $\beta_{11} r^{-1}=1+I$ for $I \in \mathcal{L}^{(1, \infty)}$.

$$
\begin{aligned}
& \operatorname{det}_{\omega}^{\frac{1}{\hbar}}\left(\gamma_{11} q^{-1}\left(1+q I q^{-1}\right)\right) \operatorname{Tr}_{\omega}\left(A_{i} \gamma_{21} q^{-1}+A_{i} \gamma_{21} I q^{-1}\right) \operatorname{Tr}_{\omega}\left(\left(B_{j} \gamma_{21} q^{-1}+B_{j} \gamma_{21} I q^{-1}\right)\right. \\
& \left.\quad \times\left(B_{k} \gamma_{21} q^{-1}+B_{k} \gamma_{21} I q^{-1}\right)\right) \\
& \left.\quad=\operatorname{det}_{\omega}\left(\gamma_{11} q^{-1}\left(1+q I q^{-1}\right)\right) \operatorname{Tr}_{\omega}\left(A_{i} \gamma_{21} q^{-1}\right) \operatorname{Tr}_{\omega}\left(B_{j} \gamma_{21} q^{-1} B_{k} \gamma_{21} q^{-1}\right)\right)
\end{aligned}
$$

In the last line we have used $\mathcal{L}^{(1, \infty)} \mathcal{L}^{(2, \infty)} \in \mathcal{L}^{1}$ and the Dixmier trace vanishes on them. As a result we get,

$$
\exp \frac{1}{\hbar}\left(\operatorname{Tr}_{\omega}\left(\gamma_{11} q^{-1}-1\right)+\operatorname{Tr}_{\omega}\left(\beta_{11} r^{-1}-1\right)\right) \operatorname{Tr}_{\omega}\left(A_{i} \gamma_{21} q^{-1}\right) \operatorname{Tr}_{\omega}\left(B_{j} \gamma_{21} q^{-1} B_{k} \gamma_{21} q^{-1}\right)
$$


where we use, $(1+I)\left(1+q I q^{-1}\right)=1+I+q J q^{-1}+I q J q^{-1}$ and the last term is zero inside the Dixmier trace for $\gamma_{11} q^{-1}=1+I, I \in \mathcal{L}^{(1, \infty)}$. This is the equivariance condition we want. We can take a product over the trace part only, and this will not change the result.

We need to check that when we reduce the wave function onto the "small" orbit, that is to the subgorup $\tilde{G}_{1+}^{(0)}$, the resulting wave functions are just constant. This is necessary for consistency with the classical Poisson bracket calculation as we will see. Let us note the following, in fact our system is invariant under a larger symmetry. This will be discussed in the next section, and we will see that the correct phase space is smaller.

One can see that we can compose a product wave function; in general we define, as in in the case of $D_{1+}$,

$$
\operatorname{det}_{\omega}^{\frac{1}{\hbar}}\left(\gamma_{11} q^{-1}\right) \prod_{i, j, k} \operatorname{Tr}_{\omega}\left(A_{i} \gamma_{21} q^{-1}\right) \operatorname{Tr}_{\omega}\left(B_{j} \gamma_{21} q^{-1} B_{k} \gamma_{21} q^{-1}\right)
$$

These and their various superpositions are the most general wave functions we can construct. The next step is to complete these set of wave functions with respect to an inner product to get the quantum Hilbert space. [2 Notice that still there could be an integrality condition on $\hbar$, due to the fact that the first Chern class of the line bundle corresponding to the representation $\rho$ should be in $H^{2}\left(G r_{1+}, \mathbf{Z}\right)$.

Let us note that the form of the wave function for the Disc and the Grassmanian are quite the same-this is unlike the previous case studied in [21]. We can in fact set up a one-to-one correspondence between the elements of the two Quantum Hilbert spaces; for any choices of $A_{i} \in \mathcal{L}^{(2, \infty)}$, and $B_{j}, B_{k}$, which are operators in $B\left(\mathcal{H}_{-}, \mathcal{H}_{+}\right)$we have a wave function on the Disc and on The Grassmanian. This suggests in some natural sense a boson-fermion correspondence, if we think of the Disc corresponding to a bosonic and the Grassmanian to a fermionic system. Of course this correspondence is only at a formal level, since we only have a set theoretical relation between the two Quantum Hilbert spaces. One also has to check the inner products, to make sure that the linearly independent choices are mapped to each other in the same manner. This seems to be a reasonable expectation, its physical meaning is not so obvious to the author; it maybe due to the fact that we have thrown away a large part of the phase space, which may have a lot of physical information about the system. We will see some further evidence of the equivalence of these two systems in this quantization scheme, when we look at these problems in a different way in the next section. ${ }^{0}$

\footnotetext{
${ }^{12}$ Notice that in our previous work [21] we could have used the trace class operators for the mixing of $\gamma_{11}$ and $\gamma_{21}$, not only the finite rank ones. Various superpositions of the wave functions described in that work will lead to a similar general form given here with a finite rank matrix used for the mixing. The finite rank operators are dense in the trace class and the determinant uses the ordinary trace. We can extend the wave functions in that case to the one with mixing elements in the trace class operators. This should actually be done by using the inner product in the quantum Hilbert space, but we expect some dominance property with respect to the parameters. In fact the above claim is known to be true, hence there is no loss of generality in that case.

13 there is larger stability subgroup as we will see in the next section and it seems to suggest a simpler solution.
} 
The above wave functions carry a representation of the group $\tilde{G}_{1+}$ which comes from the left action;

$$
\mathrm{r}\left(\gamma^{\prime}, q^{\prime}\right) \psi(\gamma, q)=\psi\left(\gamma^{\prime-1} \gamma, q^{-1} q^{\prime}\right)
$$

This group action is well-defined. We give a proof for completeness: let us denote the inverse element acting from the left by $(\lambda, r) \in \tilde{G}_{1+}$. For simplicity we drop the products and compute the following expression:

$$
\operatorname{det}_{\omega}^{\frac{1}{\hbar}}\left((\lambda \gamma)_{11} q^{-1} r^{-1}\right) \operatorname{Tr}_{\omega}\left(A_{i}(\lambda \gamma)_{21} q^{-1} r^{-1}\right) \operatorname{Tr}_{\omega}\left(B_{j}(\lambda \gamma)_{21} q^{-1} r^{-1} B_{k}(\lambda \gamma)_{21} q^{-1} r^{-1}\right)
$$

We expand the products;

$$
\begin{aligned}
& \operatorname{det}_{\omega}^{\frac{1}{\hbar}}\left(\left(\lambda_{11} \gamma_{11}+\lambda_{12} \gamma_{21}\right) q^{-1} r^{-1}\right) \operatorname{Tr}_{\omega}\left(A_{i}\left(\lambda_{21} \gamma_{11}+\lambda_{22} \gamma_{21}\right) q^{-1} r^{-1}\right) \\
& \left.\quad \times \operatorname{Tr}_{\omega}\left(B_{j}\left(\lambda_{21} \gamma_{11}+\lambda_{22} \gamma_{21}\right) q^{-1} r^{-1} B_{k}\left(\lambda_{21} \gamma_{11}+\lambda_{22} \gamma_{21}\right) q^{-1} r^{-1}\right)\right) \\
& =\operatorname{det}_{\omega}^{\frac{1}{\hbar}}\left((1+I)\left(1+r J r^{-1}\right)+\lambda_{12} \gamma_{21} q^{-1} r^{-1}\right) \operatorname{Tr}_{\omega}\left(A_{i} \lambda_{21}(1+I) r^{-1}+A_{i} \lambda_{22} \gamma_{21} q^{-1} r^{-1}\right) \\
& \times \operatorname{Tr}_{\omega}\left[B_{j} \lambda_{21}(1+I) r^{-1}+B_{j} \lambda_{22} \gamma_{21} q^{-1} r^{-1}\right]\left[B_{k} \lambda_{21}(1+I) r^{-1}+B_{k} \lambda_{22} \gamma_{21} q^{-1} r^{-1}\right] \\
& =\operatorname{det}_{\omega}^{\frac{1}{\hbar}}\left(1+I+J+\lambda_{12} \gamma_{21} q^{-1} r^{-1}\right) \times \operatorname{Tr}_{\omega}\left(A_{i}\left(\lambda_{21} r^{-1}+\lambda_{22} \gamma_{21}\right) q^{-1} r^{-1}\right. \\
& \times \operatorname{Tr}_{\omega}\left[B_{j} \lambda_{21} r^{-1}+B_{j} \lambda_{22} \gamma_{21} q^{-1} r^{-1}\right]\left[B_{k} \lambda_{21} r^{-1}+B_{k} \lambda_{22} \gamma_{21} q^{-1} r^{-1}\right]
\end{aligned}
$$

where we use $\gamma_{11} q^{-1}=1+I$ and $\lambda_{11} r^{-1}=1+J$. Notice also that we used the vanishing of Dixmier trace whenever the resulting multiplication is in the trace class operators-and this is the case for example for products of the kind $\lambda_{21} I$ and for other similar terms. All the terms in the above expression are actually in the ideal $\mathcal{L}^{(1, \infty)}$ except 1 , hence we have the result well-defined.

Clearly the action of a group element on a general wave function follows directly from this result. It follows immediately from the above formula that when we restrict to the subspace $\tilde{G}_{1+}^{(0)}$ for the wave function elements and the group element multiplying from the left, the expression inside the determinant gives us 1 . This shows that the representation is trivial.

For completeness we will prove that we have a true representation of the group $\tilde{G}_{1+}$ on these space of wave functions. This is a technical step, and can be skipped. We will compare the action of the group elements and show that

$$
\psi(\lambda(\sigma \gamma), r(s q))=\psi((\lambda \sigma) \gamma,(r s) q),
$$

hence when we apply the left multiplication on the left this gives us a representation. we have the group action by $(\lambda, r)$ given by the above formula that we have already calculated;

$$
\begin{aligned}
& \operatorname{det}_{\omega}^{\frac{1}{\hbar}}\left(\lambda_{11} r^{-1}\right) \operatorname{det}^{\frac{1}{\hbar}}\left(\gamma_{11} q^{-1}+\lambda_{12} \gamma_{21} q^{-1} r^{-1}\right) \operatorname{Tr}_{\omega} A_{i}\left(\lambda_{21} r^{-1}+\lambda_{22} \gamma_{21} q^{-1} r^{-1}\right) \\
& \quad \times \operatorname{Tr}_{\omega}\left(B_{j}\left(\lambda_{21} r^{-1}+\lambda_{22} \gamma_{21} q^{-1} r^{-1}\right) B_{k}\left(\lambda_{21} r^{-1}+\lambda_{22} \gamma_{21} q^{-1} r^{-1}\right)\right)
\end{aligned}
$$

We can act with the element $(\sigma, s)$ on this; it is better to break the terms into separate parts:

$$
\operatorname{det}^{\frac{1}{\hbar}}\left(\lambda_{11} r^{-1}\right) \operatorname{det}_{\omega}^{\frac{1}{\hbar}}\left(\left(\sigma_{11} \gamma_{11}+\sigma_{12} \gamma_{21}\right) q^{-1} s^{-1}+\lambda_{12}\left(\sigma_{21} \gamma_{11}+\sigma_{22} \gamma_{21}\right) q^{-1}(r s)^{-1}\right)
$$




$$
\operatorname{det}_{\omega}^{\frac{1}{\hbar}}\left(\lambda_{11} r^{-1}\right) \operatorname{det}_{\omega}^{\frac{1}{\hbar}}\left(\sigma_{11} s^{-1}\right) \operatorname{det}_{\omega}^{\frac{1}{\hbar}}\left(\gamma_{11} q^{-1}+\sigma_{12} \gamma_{21} q^{-1} s^{-1}+\lambda_{12} \sigma_{21}(r s)^{-1}+\lambda_{12} \sigma_{22} \gamma_{21} q^{-1}(r s)^{-1}\right) .
$$

We compare this with the action of the group element $(\lambda \sigma, r s)$ on the same wave function;

$$
\begin{aligned}
& \operatorname{det}_{\omega}^{\frac{1}{\hbar}}\left((\lambda \sigma)_{11}(r s)^{-1}\right) \operatorname{det}^{\frac{1}{\hbar}}\left(\gamma_{11} q^{-1}+(\lambda \sigma)_{12} \gamma_{21} q^{-1}(r s)^{-1}\right)= \\
& \operatorname{det}_{\omega}^{\frac{1}{\hbar}}\left(\lambda_{11} \sigma_{11} s^{-1} r^{-1}+\lambda_{12} \sigma_{21} s^{-1} r^{-1}\right) \operatorname{det}_{\omega}^{\frac{1}{\hbar}}\left(\gamma_{11} q^{-1}+\lambda_{11} \sigma_{12} \gamma_{21} q^{-1} s^{-1} r^{-1}+\lambda_{12} \sigma_{22} \gamma_{21} q^{-1}(r s)^{-1}\right) \\
& \quad=\operatorname{det}_{\omega}^{\frac{1}{\hbar}}\left(\lambda_{11} r^{-1}\right) \operatorname{det}_{\omega}^{\frac{1}{\hbar}}\left(\sigma_{11} s^{-1}\right) \operatorname{det}_{\omega}^{\frac{1}{\hbar}}\left(\gamma_{11} q^{-1}+\lambda_{12} \sigma_{21}(r s)^{-1}+\sigma_{12} \gamma_{21} q^{-1} s^{-1}+\lambda_{12} \sigma_{22} \gamma_{21} q^{-1}(r s)^{-1}\right) .
\end{aligned}
$$

These two expressions are the same, hence we have a group action on this part of the wave function.

Let us check the next term;

$$
\begin{aligned}
& \operatorname{Tr}_{\omega}\left(A_{i}\left(\lambda_{21} r^{-1}+\lambda_{22}\left(\sigma_{21} \gamma_{11}+\sigma_{22} \gamma_{21}\right) q^{-1}(r s)^{-1}\right)=\right. \\
& \operatorname{Tr}_{\omega}\left(A_{i}\left(\lambda_{21} r^{-1}+\lambda_{22} \sigma_{21}(r s)^{-1}+\lambda_{22} \sigma_{22} \gamma_{21} q^{-1}(r s)^{-1}\right) .\right.
\end{aligned}
$$

Let us compare this with the direct application of the product;

$$
\begin{aligned}
& \operatorname{Tr}_{\omega}\left(A_{i}\left((\lambda \sigma)_{21}(r s)^{-1}+(\lambda \sigma)_{22} \gamma_{21} q^{-1}(r s)^{-1}\right)=\right. \\
& \operatorname{Tr}_{\omega}\left(A_{i}\left(\lambda_{21} \sigma_{11} s^{-1} r^{-1}++\lambda_{22} \sigma_{21}(r s)^{-1}+\lambda_{21} \sigma_{12} \gamma_{21} q^{-1}(r s)^{-1}+\lambda_{22} \sigma_{22} \gamma_{21} q^{-1}(r s)^{-1}\right)\right. \\
& =\operatorname{Tr}_{\omega}\left(A_{i}\left(\lambda_{21} r^{-1}+\lambda_{22} \sigma_{21}(r s)^{-1}+\lambda_{22} \sigma_{22} \gamma_{21} q^{-1}(r s)^{-1}\right) .\right.
\end{aligned}
$$

These two expressions are the same. Let us look at the last type of term;

$$
\begin{aligned}
& \operatorname{Tr}_{\omega}\left(B_{j}\left(\lambda_{21} r^{-1}+\lambda_{22}\left(\sigma_{21} \gamma_{11}+\sigma_{22} \gamma_{21}\right) q^{-1}(r s)^{-1}\right) B_{k}\left(\lambda_{21} r^{-1}+\lambda_{22}\left(\sigma_{21} \gamma_{11}+\sigma_{22} \gamma_{21}\right) q^{-1}(r s)^{-1}\right)\right) \\
= & \operatorname{Tr}_{\omega}\left(B_{j}\left(\lambda_{21} r^{-1}+\lambda_{22} \sigma_{21}(r s)^{-1}+\lambda_{22} \sigma_{22} \gamma_{21} q^{-1}(r s)^{-1}\right)\right. \\
& \left.B_{k}\left(\lambda_{21} r^{-1}+\lambda_{22} \sigma_{21}(r s)^{-1}+\lambda_{22} \sigma_{22} \gamma_{21} q^{-1}(r s)^{-1}\right)\right) .
\end{aligned}
$$

If we look at the action of the product:

$$
\begin{gathered}
\left.\left.\operatorname{Tr}_{\omega}\left(B_{j}(\lambda \sigma)_{21}(r s)^{-1}+(\lambda \sigma)_{22} \gamma_{21} q^{-1}(r s)^{-1}\right) B_{k}(\lambda \sigma)_{21}(r s)^{-1}+(\lambda \sigma)_{22} \gamma_{21} q^{-1}(r s)^{-1}\right)\right) \\
=\operatorname{Tr}_{\omega}\left(B_{j}\left(\lambda_{21} r^{-1}+\lambda_{22} \sigma_{21}(r s)^{-1}+\left(\lambda_{21} \sigma_{12}+\lambda_{22} \sigma_{22}\right) \gamma_{21} q^{-1}(r s)^{-1}\right)\right. \\
\left.B_{k}\left(\lambda_{21} r^{-1}+\lambda_{22} \sigma_{21}(r s)^{-1}+\left(\lambda_{21} \sigma_{12}+\lambda_{22} \sigma_{22}\right) \gamma_{21} q^{-1}(r s)^{-1}\right)\right) \\
=\operatorname{Tr}_{\omega}\left(B_{j}\left(\lambda_{21} r^{-1}+\lambda_{22} \sigma_{21}(r s)^{-1}+\lambda_{22} \sigma_{22} \gamma_{21} q^{-1}(r s)^{-1}\right)\right. \\
\left.B_{k}\left(\lambda_{21} r^{-1}+\lambda_{22} \sigma_{21}(r s)^{-1}+\lambda_{22} \sigma_{22} \gamma_{21} q^{-1}(r s)^{-1}\right)\right),
\end{gathered}
$$

where we used the vanishing of Dixmier trace on $\mathcal{L}^{1}$ and some rearrangements on all the above calculations. We see that this term also respects the group action.

The above representation factors through the subgroup which corresponds to the elements $s$ of $G L\left(\mathcal{H}_{-}\right)$, with $\operatorname{det}_{\omega}(s)$ well-defined. These are the elements in the subgroup, $s \in$ $1+\mathcal{L}^{(1, \infty)}$. Notice that for a fixed element $\gamma$, the freedom we have to choose different $q$ 's which satisfy the determinant condition, is isomorphic to $G L^{1+}=\left\{q \in G L\left(\mathcal{H}_{-}\right) \mid q=\right.$ 
$\left.1+\mathcal{L}^{(1, \infty)}\right\}$ Although we could not find a way to reduce it to this group everywhere. Hence our representations can actually be reduced to the representations of another group, which is a central extension of the group $G L_{1+}$. The extension is trivial on the subgroup $G L_{1+}^{(0)}$, this can be seen by noticing that $s \in 1+\left(\mathcal{L}^{(1, \infty)}\right)^{(0)}$ will give us $\operatorname{det}_{\omega}(s)=1$. We can construct a commutative diagram as we have done in [21], to show that this gives us a representation of a central extension of the group $G L_{1+}$. This is more transparent if we look in the Lie algebra level, and also this gives us a chance to compare the central term we have in the case of moment maps. The Lie algebra we need to consider is clearly $\left\{(u, r) \mid r \in \operatorname{End}\left(\mathcal{H}_{-}\right) \quad u_{11}-r \in\right.$ $\left.\mathcal{L}^{(1, \infty)}\right\}$. One can see that the Lie bracket $[(u, r),(v, s)]=([u, v],[r, s])$ is well-defined, and this is the infinitesimal form of the group $\tilde{G}_{1+}$. One can see immediately that the set of possible $r$ 's is isomorphic to the set of $r \in G L\left(\mathcal{H}_{-}\right)$such that $r \in \mathcal{L}^{(1, \infty)}$. We can construct a central extension of the original Lie algebra by using, $(u, r) \mapsto\left(u, \operatorname{Tr}_{\omega}\left(u_{11}-r\right)\right)$. We give the trivial Lie bracket to the complex numbers. Under this map, the commutator goes to

$$
\left([u, v], \operatorname{Tr}_{\omega}\left([u, v]_{11}-[r, s]\right)\right)=\left([u, v], \operatorname{Tr}_{\omega}^{\epsilon}([\epsilon, u] v)\right)
$$

where we used

$$
\begin{aligned}
& \operatorname{Tr}_{\omega}\left(u_{11} v_{11}-v_{11} u_{11}+u_{12} v_{21}-v_{12} u_{21}-[r, s]\right) \\
& =\operatorname{Tr}_{\omega}\left(u_{11} v_{11}-r s-v_{11} u_{11}+s r\right)+\operatorname{Tr}_{\omega}\left(u_{12} v_{21}-v_{12} u_{21}\right) \\
& =\operatorname{Tr}_{\omega}\left(\left(u_{11}-r\right) v_{11}+r\left(v_{11}-s\right)\right)-\operatorname{Tr}_{\omega}\left(v_{11}\left(u_{11}-r\right)+\left(v_{11}-s\right) r\right)+\operatorname{Tr}_{\omega}^{\epsilon}([\epsilon, u] v) \\
& =\operatorname{Tr}_{\omega}^{\epsilon}([\epsilon, u] v)
\end{aligned}
$$

since all the terms, as grouped, are in $\mathcal{L}^{(1, \infty)}$ and we use the Dixmier trace properties. Note that this is the same central extension in the case of moment maps that we have been using. We will show by an explicit calculation that our representations can be reduced to representations of this algebra; let us first compute the infinitesimal action, for simplicity we drop the product signs:

$$
\mathcal{L}_{(u, r)} \operatorname{det}_{\omega}^{\frac{1}{\hbar}}\left(\gamma_{11} q^{-1}\right) \operatorname{Tr}_{\omega}\left(A_{i} \gamma_{21} q^{-1}\right) \operatorname{Tr}_{\omega}\left(B_{j} \gamma_{21} q^{-1} B_{k} \gamma_{21} q^{-1}\right)
$$

It is again easier to use the derivation property of the Lie derivative and compute individual terms.

$$
\begin{aligned}
& \mathcal{L}_{(u, r)} \operatorname{det}_{\omega}^{\frac{1}{\hbar}}\left(\gamma_{11} q^{-1}\right)=\frac{-1}{\hbar} \operatorname{Tr}_{\omega}\left(\left(u_{11} \gamma_{11}+u_{12} \gamma_{21}\right) q^{-1}-\gamma_{11} q^{-1} r\right) \operatorname{det}_{\omega}^{\frac{1}{\hbar}}\left(\gamma_{11} q^{-1}\right) \\
& =\quad\left(-\frac{1}{\hbar} \operatorname{Tr}_{\omega}\left(u_{11}-r\right)-\frac{1}{\hbar} \operatorname{Tr}_{\omega}\left(u_{12} \gamma_{21} q^{-1}\right)\right) \operatorname{det}_{\omega}^{\frac{1}{\hbar}}\left(\gamma_{11} q^{-1}\right) .
\end{aligned}
$$

In a similar way we get;

$$
\mathcal{L}_{(u, r)} \operatorname{Tr}_{\omega}\left(A_{i} \gamma_{21} q^{-1}\right)=\operatorname{Tr}_{\omega}\left(A_{i}\left(u_{21}+u_{22} \gamma_{21} q^{-1}-\gamma_{21} q^{-1} r\right)\right)
$$

and

$$
\mathcal{L}_{(u, r)} \operatorname{Tr}_{\omega}\left(B_{j} \gamma_{21} q^{-1} B_{k} \gamma_{21} q^{-1}\right)
$$




$$
=\operatorname{Tr}_{\omega}\left(B_{j}\left(u_{21}+u_{22} \gamma_{21} q^{-1}-\gamma_{21} q^{-1} r\right) B_{k}\left(u_{21}+u_{22} \gamma_{21} q^{-1}-\gamma_{21} q^{-1} r\right)\right) .
$$

We define a new representation, and using the above expressions check that it is in fact independent of the choice of the Lie algebra element $r$;

$$
\hat{\mathrm{r}}[(u, r)]=\mathcal{L}_{(u, r)}+\frac{1}{\hbar} \operatorname{Tr}_{\omega}\left(u_{11}-r\right),
$$

acting on the same set of wave functions. Using the above expression, we compute the action of the Lie algebra element $(u, r+s)$, where $s \in \mathcal{L}^{(1, \infty)}$ and this is the freedom we have. It is again simpler to check this on each basic piece;

$$
\begin{aligned}
\left(\hat{\mathrm{r}}[(u, r+s)] \quad \operatorname{det}^{\frac{1}{\hbar}}\left(\gamma_{11} q^{-1}\right)\right) \phi\left(A_{i}, B_{j}, B_{k}, \gamma, q\right) \\
\quad=\left[\mathcal{L}_{(u, r+s)}+\frac{1}{\hbar} \operatorname{Tr}_{\omega}\left(u_{11}-(r+s)\right)\right] \operatorname{det}^{\frac{1}{\hbar}}\left(\gamma_{11} q^{-1}\right) \phi\left(A_{i}, B_{j}, B_{k}, \gamma, q\right) \\
\quad=-\frac{1}{\hbar} \operatorname{Tr}_{\omega}\left(u_{12} \gamma_{21} q^{-1}\right) \operatorname{det}_{\omega}^{\frac{1}{\hbar}}\left(\gamma_{11} q^{-1}\right) \phi\left(A_{i}, B_{j}, B_{k}, \gamma, q\right) .
\end{aligned}
$$

For the other terms we only use the Lie derivative part since the scalar part is used in the above expression already;

$$
\begin{aligned}
& \mathcal{L}_{(u, r+s)} \operatorname{Tr}_{\omega}\left(A_{i} \gamma_{21} q^{-1}\right)=\operatorname{Tr}_{\omega}\left(A_{i}\left(u_{21}+u_{22} \gamma_{21} q^{-1}-\gamma_{21} q^{-1}(r+s)\right)\right) \\
= & \operatorname{Tr}_{\omega}\left(A_{i}\left(u_{21}+u_{22} \gamma_{21} q^{-1}-A_{i} \gamma_{21} q^{-1} r\right)=\mathcal{L}_{(u, r)} \operatorname{Tr}_{\omega}\left(A_{i} \gamma_{21} q^{-1}\right),\right.
\end{aligned}
$$

by using the fact that everytime $s$ is multiplied with an element, the resulting term is in the ideal of trace class operators, and the Dixmier trace vanishes on them. The other term,

$$
\mathcal{L}_{(u, r+s)} \operatorname{Tr}_{\omega}\left(B_{j} \gamma_{21} q^{-1} B_{k} \gamma_{21} q^{-1}\right),
$$

can be shown to be independent of $s$ by using the same reasoning as above. Hence we can denote the representation we have as $\hat{\mathrm{r}}(u)$.

If we compute the commutator,

$$
(\hat{\mathrm{r}}(u) \hat{\mathrm{r}}(v)-\hat{\mathrm{r}}(v) \hat{\mathrm{r}}(v)) \psi(\gamma, q)=\left[\hat{\mathrm{r}}([v, u])-\frac{1}{\hbar} \operatorname{Tr}_{\omega}\left([u, v]_{11}-[r, p]\right)\right] \psi(\gamma, q) .
$$

The last term is independent of the choices of $r, p$ and equal to $-\frac{1}{\hbar} \operatorname{Tr}_{\omega}^{\epsilon}[\epsilon, u] v$ as we have seen before.

Hence the representations that we have obtained can be reduced to the representations of the central extension of the Lie group $U_{1+}(\mathcal{H})$. This is the quantization of our classical system, it may not be possible to express the central term corresponding to the group in this form, since the extension may have a topological twist in general. We are not able to answer this question, although the discussion in the last section hints that the correct phase space is topologically trivial. This will imply that the central extension actually comes from a central term, globably defined. 


\section{Flat Geometry and Quantization}

In this section we will introduce a classical system which appears to be unrelated at first sight. This point of view was suggested by Rajeev in our discussions. We consider the set of elements $\bar{Z}$ such that they belong to the following quotient space $\mathcal{L}^{(2, \infty)}\left(\mathcal{H}_{+}, \mathcal{H}_{-}\right) /\left(\mathcal{L}^{(2, \infty)}\left(\mathcal{H}_{+}, \mathcal{H}_{-}\right)\right)^{(0)}$ (equivalence classes of $Z: \mathcal{H}_{+} \rightarrow \mathcal{H}_{-}$and $Z \in \mathcal{L}^{(2, \infty)}\left(\mathcal{H}_{+}, \mathcal{H}_{-}\right)$under $\left.Z-Z^{\prime} \in\left(\mathcal{L}^{(2, \infty)}\left(\mathcal{H}_{+}, \mathcal{H}_{-}\right)\right)^{(0)}\right)$. There is a natural quotient norm;

$$
\|\bar{Z}\|=\inf _{Z_{0} \in\left(\mathcal{L}^{(2, \infty)}\right)(0)}\left\|Z+Z_{0}\right\|_{\mathcal{L}^{(2, \infty)}}
$$

where $Z$ is a representative in the equivalence class of $\bar{Z}$.

There is a natural product from $\mathcal{L}^{(2, \infty)} \times \mathcal{L}^{(2, \infty)} \rightarrow \mathcal{L}^{(1, \infty)}$, and this reduces to the quotients; $\mathcal{L}^{(2, \infty)} /\left(\mathcal{L}^{(2, \infty)}\right)^{(0)} \times \mathcal{L}^{(2, \infty)} /\left(\mathcal{L}^{(2, \infty)}\right)^{(0)} \rightarrow \mathcal{L}^{(1, \infty)} /\left(\mathcal{L}^{(1, \infty)}\right)^{(0)}$ given by $\bar{Z} \bar{Z}^{\prime}=\overline{Z Z^{\prime}}$ The natural product $B(\mathcal{H}) \times \mathcal{L}^{(2, \infty)} \rightarrow \mathcal{L}^{(2, \infty)}$ also descends to the quotient; $B(\mathcal{H}) \times$ $\left(\mathcal{L}^{(2, \infty)} /\left(\mathcal{L}^{(2, \infty)}\right)^{(0)}\right) \rightarrow \mathcal{L}^{(2, \infty)} /\left(\mathcal{L}^{(2, \infty)}\right)^{(0)}$.

The Dixmier trace is nondegenerate on this quotient space. We will use this important fact to introduce an obvious symplectic form.

$$
\tilde{\Omega}_{\omega}=i \operatorname{Tr}_{\omega} d \bar{Z} \wedge d \bar{Z}^{\dagger}
$$

This flat geometry has a simple symmetry group [4]; given by rotations and translations. Due to the quotient we can allow for slight deviations from unitary transformations and write a general transformation as

$$
\bar{Z} \mapsto \overline{\overline{e Z f^{-1}}+l},
$$

where $e \in G L\left(\mathcal{H}_{-}\right), f \in G L\left(\mathcal{H}_{+}\right)$such that $e^{\dagger} e-1, f^{\dagger} f-1 \in K(\mathcal{H})$, and $l \in \mathcal{L}^{(2, \infty)}$. One can check that this is in fact a group under the obvious composition law, which we call as the affine group, $\mathcal{A}_{1+}$. This action is well-defined and transitive. One can immediately check that the group action preserves the symplectic form due to the extra conditions we have;

$$
\tilde{\Omega}_{\omega}=i \operatorname{Tr}_{\omega} e d \bar{Z} f^{-1} \wedge f^{-1 \dagger} d \bar{Z}^{\dagger} e^{\dagger}=i \operatorname{Tr}_{\omega}\left(e^{\dagger} e\right) d \bar{Z} \wedge\left(f^{\dagger} f\right)^{-1} d \bar{Z}^{\dagger}=\tilde{\Omega}_{\omega},
$$

using the fact that the porduct $e^{\dagger} e d \bar{Z} \approx d \bar{Z}$ and the same for $f$ (see Appendix for a proof).

It is again natural to find the moment maps generating this action. We can find them using the infinitesimal form of the group action; $\tilde{V}_{(e, f, l)}(\bar{Z})=\overline{\alpha Z}-\overline{Z \delta}+\bar{\beta}$ (here, we denote the Lie algebra elements by the same letter $\alpha, \beta, \delta$, but they now satisfy $\alpha^{\dagger}+\alpha=1+K, \delta^{\dagger}+\delta=$ $1+K$ where $K$ is a compact operator, and $\bar{\beta} \in \mathcal{L}^{(2, \infty)} /\left(\mathcal{L}^{(2, \infty)}\right)^{(0)}$, yet we denote the moment maps by $F_{(e, f, l)}$ to imply that they are coming from the affine action. we hope that this does not cause too much confusion). Hence,

$$
F_{(e, f, l)}=i \operatorname{Tr}_{\omega}\left(\alpha \bar{Z} \bar{Z}^{\dagger}-\bar{Z} \delta \bar{Z}^{\dagger}+\bar{\beta} \bar{Z}^{\dagger}+\bar{Z} \bar{\beta}^{\dagger}\right)
$$

\footnotetext{
${ }^{14}$ This may not be the most general action, but it is the obvious one.
} 
If we compute $\left\{F_{\left(e_{1}, f_{1}, l_{1}\right)}, F_{\left(e_{2}, f_{2}, l_{2}\right)}\right\}$ we will see that there is a central term. Since we will do this calculation below to make connection with the previous section, we postpone the result.

Geometric quantization gives us immediately the following general set of wave functions;

$$
\Psi\left(\bar{Z}, \bar{Z}^{\dagger}\right)=e^{-\frac{1}{\bar{\hbar}} \operatorname{Tr}_{\omega} \bar{Z} \bar{Z}^{\dagger}} \prod_{i, j, k} \operatorname{Tr}_{\omega}\left(\bar{A}_{i} \bar{Z}\right) \operatorname{Tr}_{\omega}\left(B_{j} \bar{Z} B_{k} \bar{Z}\right)
$$

where $\bar{A}_{i} \in \mathcal{L}^{(2, \infty)}$ and $B_{j, k}$ are bounded. Naturally, this set of wave functions carry a representation of the central extension of the above group action, via the same type of operators we have found before ${ }^{\mathrm{T}}$;

$$
\hat{F} \psi(\bar{Z})=\left(\mathcal{L}_{(\alpha, \delta, \beta)}-\frac{1}{\hbar} \operatorname{Tr}_{\omega}\left(\bar{\beta}^{\dagger} \bar{Z}\right)\right) \psi(\bar{Z})
$$

This system has an interesting connection to our discussions on the previous section. Let us recall the Disc case. One can recover the Symplectic form for the Disc using the following Kähler potential, $i \operatorname{Tr}_{\omega} \log \left(1-Z Z^{\dagger}\right)$, just as in the finite dimensional case. Let us expand the Kähler form, and use the properties of the Dixmier trace. We see that the result is a simple expresion; $i \operatorname{Tr}_{\omega} Z Z^{\dagger}$. This is the result for a flat system, except for degeneracies. If we look at the quotient, as above, the result is the same as the Kähler potential of the above system.

We can also apply the quotient homomorphism to our pseudo-unitary group action; this gives,

$$
\bar{Z} \mapsto \overline{a Z d^{-1}}+\overline{b d^{-1}}
$$

Let us show that the group property is preserved under this mapping;

$$
\begin{aligned}
\overline{g_{2} \circ\left(g_{1} \circ Z\right)} & =\frac{a_{2}\left(a_{1} \bar{Z} d_{1}^{-1}+\overline{b_{1} d_{1}^{-1}}\right) d_{2}^{-1}+\overline{b_{2} d_{2}^{-1}}=a_{2} a_{1} \bar{Z}\left(d_{2} d_{1}\right)^{-1}+a_{2} \overline{b_{1}\left(d_{2} d_{1}\right)^{-1}}=}{}=\overline{\left(a_{2} a_{1}+b_{2} b_{1}\right) Z\left(d_{2} d_{1}+c_{2} c_{1}\right)^{-1}}+\overline{\left(a_{2} b_{1}+b_{2} d_{1}\right)\left(d_{2} d_{1}+c_{2} c_{1}\right)^{-1}}=\overline{\left(g_{2} g_{1}\right) \circ Z}
\end{aligned}
$$

This gives us an embedding of $U_{1+}\left(\mathcal{H}_{-}, \mathcal{H}_{+}\right)$into the affine group. Another interesting point is to look at the moment maps; and expand $\operatorname{Tr}_{\omega} u(\Phi-\epsilon)$ in the variable $Z$, by using the expression of $\Phi$ in terms of $Z$. The properties of the Dixmier trace can be used to see that most of the terms vanish; the result is the same as the moment maps of the flat system:

$$
f_{u}=i \operatorname{Tr}_{\omega}\left(\alpha Z Z^{\dagger}-Z \delta Z^{\dagger}+\beta Z+Z^{\dagger} \beta^{\dagger}\right)
$$

Of course, it is natural to go to the quotient again, and we get $F_{u}=F_{\left(a, d, b d^{-1}\right)}$. We can now compute to see the Poisson bracket of these two moment maps, using the flat Poisson bracket;

$$
\begin{aligned}
\left\{F_{u}, F_{v}\right\} & =i \operatorname{Tr}_{\omega}\left(\left[\alpha_{1}, \alpha_{2}\right] \bar{Z} \bar{Z}^{\dagger}-\bar{Z}\left[\delta_{1}, \delta_{2}\right] \bar{Z}^{\dagger}+\left(\alpha_{2} \beta_{1}-\alpha_{1} \beta_{2}+\beta_{1} \delta_{2}-\beta_{2} \delta_{1}\right) \bar{Z}^{\dagger}\right. \\
& \left.+\bar{Z}\left(\alpha_{2} \beta_{1}-\alpha_{1} \beta_{2}+\beta_{1} \delta_{2}-\beta_{2} \delta_{1}\right)^{\dagger}\right)+i \operatorname{Tr}_{\omega}\left(\bar{\beta}_{1} \beta_{2}^{\dagger}-\beta_{2} \beta_{1}^{\dagger}\right) .
\end{aligned}
$$

\footnotetext{
${ }^{15}$ We skip a detailed derivation of this formula, but the reader can verify it by using standard geometric quantization.
} 
One can verify that the last term is a central term which is equal to the central term we have found before.

The above set of wave functions are equivalent to the wave functions on the disc and they carry the same representation of the central extension of the quotient group. This shows that the system we studied without the reduction can be put into a slightly bigger flat system.

The same question then arises for the Grassmannian. Its coordinatization will show that in each coordinate neighborhood, the symplectic form is given by the flat one, and similarly the moment functions will look like the flat geometry. Certainly, the quotient point of view, using $U_{1+} /\left(U_{1+}\right)^{(0)}$, implies that there is a similar simplification. Now we will try to present an alternative point of view in the Grassmanian which keeps the complex structure. Consider the following subgroup;

$$
\tilde{G}_{(1+, 0)}=\left\{(g, q) \mid g \in\left(\begin{array}{cc}
\mathcal{B} & \mathcal{L}^{(2, \infty)} \\
\left(\mathcal{L}^{(2, \infty)}\right)^{(0)} & \mathcal{B}
\end{array}\right)\right\} .
$$

This is a closed subgroup, hence the quotient is a holomorphic manifold. Notice that the representation we have introduced for the subgroup $\tilde{B}_{1+}$, actually extend to a representation of this larger group:

$$
\operatorname{det}_{\omega}^{\frac{1}{\hbar}}\left((\gamma \lambda)_{11} s^{-1} q^{-1}\right)=\operatorname{det}_{\omega}^{\frac{1}{\hbar}}\left(\gamma_{11} \lambda_{11} s^{-1} q^{-1}+\gamma_{12} \lambda_{21} s^{-1} q^{-1}\right),
$$

notice that the last term is actually zero under the Dixmier trace and the rest follows as before, showing that it is a one-dimensional holomorphic representation. The next thing is to check that the remaining part of the wave function is in fact invariant under the group $\tilde{G}_{(1+, 0)}$. Thus, we will have a line bundle on this smaller quotient, which is the physically relevant phase space. Let us only check one of the terms;

$$
\begin{aligned}
& \operatorname{Tr}_{\omega}\left(A_{i}(\gamma \lambda)_{21} s^{-1} q^{-1}\right)=\operatorname{Tr}_{\omega}\left(A_{i}\left(\gamma_{21} \lambda_{11} s^{-1} q^{-1}+\gamma_{22} \lambda_{21} s^{-1} q^{-1}\right)\right) \\
& =\operatorname{Tr}_{\omega}\left(A_{i} \gamma_{21} q^{-1}\right)
\end{aligned}
$$

by using the fact that the last term is in $\left(\mathcal{L}^{(1, \infty)}\right)^{(0)}$. Similarly for the other type of term. Hence we can consider the set of functions as the setions of a line bundle on the quotient; $\tilde{G}_{1+} / \tilde{G}_{(1+, 0)}$. Since we already know that for $B_{1+}$ the quotient cancel out the $q$ parts, for the above form also we get

$\tilde{G}_{1+} / \tilde{G}_{(1+, 0)} \approx G L_{1+} / G L_{(1+, 0)}=\left(\begin{array}{cc}\mathcal{B} & \mathcal{L}^{(2, \infty)} \\ \mathcal{L}^{(2, \infty)} & \mathcal{B}\end{array}\right) /\left(\begin{array}{cc}\mathcal{B} & \mathcal{L}^{(2, \infty)} \\ \left(\mathcal{L}^{(2, \infty)}\right)^{(0)} & \mathcal{B}\end{array}\right) \approx \mathcal{L}^{(2, \infty)} /\left(\mathcal{L}^{(2, \infty)}\right)^{(0)}$

This shows that the relevant part of the phase spaces for the Disc and the Grassmanian are of equal size. We are not able to provide a link with this and the coordinate description at the present moment. Our guess is that Grassmanian also has the same embedding into a flat system, this manifests the possible equivalence of the two systems. We hope to clarify some of these issues in a future publication. 


\section{Acknowledgements}

Most of this paper owes a lot to several conversations with S.G. Rajeev, and shows his guidance and influence. We thank J. Mickelsson for reading and suggesting corrections, and to M. Wodzicki for a series of marvelous lectures on traces, to E. Langmann for several suggestions.T- We also would like to thank J. Brodzki, P. Bongaart, K. Gawedzki, G. Landi, M. Walze, for many useful discussions. The author gratefully acknowledges the hospitality of S. Majid and his group, and the kind invitation of P. Goddard and D. Crighton to DAMTP, where most of this work done during the author's stay in Cambridge. During this project the author is supported by the EPDI grant, and we thank IHES for the excellent help and working environment provided.

\section{Appendix}

The definitions of the operator ideals will be given. Let us start with the definition of $\mathcal{L}^{(2, \infty)}$. Operator ideals contains compact operators, thus they are given by the summability properties of the singular values of the operators. If $s_{n}(A)=n$th eigenvalue of $|A|$, then we define a new norm:

$$
\|A\|_{\mathcal{L}^{(2, \infty)}}=\sup _{N} \frac{\sum_{n=1}^{N} s_{n}(A)}{\sum_{i=1}^{N} \frac{1}{n^{1 / 2}}}
$$

The set of all $A \in K(\mathcal{H})$ for which the above norm is finite is denoted by $\mathcal{L}^{(2, \infty)}$. It is a symmetrically normed ideal [12]. Since the sequence $\frac{1}{n^{1 / 2}}$ is regular, the same ideal can be defined through the asymptotic behaviour of the singular values. In fact, the set of operators in $\mathcal{L}^{(2, \infty)}$ can be defined as $A \in K(\mathcal{H})$ such that $s_{n}(A)=O\left(n^{-1 / 2}\right)$. This also gives a simple characterization of the completion of the finite rank operators inside $\mathcal{L}^{(2, \infty)}$, denoted as $\left(\mathcal{L}^{(2, \infty)}\right)^{(0)} ; A \in\left(\mathcal{L}^{(2, \infty)}\right)^{(0)}$ iff $s_{n}(A)=o\left(n^{-1 / 2}\right) .{ }^{\top 7}$ One can define the norm for $\mathcal{L}^{(1, \infty)}$ in the same way replacing the sequence $1 / n^{1 / 2}$ by $1 / n$. This is not a regular sequence so the completion of finite rank operators are given by the behaviour of the partial sums; $\sigma_{N}(A)=\sum_{n=1}^{N} s_{n}(A), \sigma_{N}(A)=O(\log N)$. If $s_{n}(B)=o\left(\frac{1}{n}\right)$, then it implies that $\sigma_{N}(B)=o(\log N)$, hence $B \in\left(\mathcal{L}^{(1, \infty)}\right)^{(0)}$. But the converse is not true.

We give a proof that $A, B \in \mathcal{L}^{(1, \infty)}$ then $A B \in \mathcal{L}^{1}$. Let us assume that the hypothesis is true. It implies that $A, B \in \mathcal{L}^{2}$ as well. But we know that $\mathcal{L}^{2} \mathcal{L}^{2} \in \mathcal{L}^{1}$, hence the result.

Next, we will prove that if $A \in \mathcal{L}^{(2, \infty)}$ and $B \in\left(\mathcal{L}^{(2, \infty)}\right)^{(0)}$ then $A B \in\left(\mathcal{L}^{(1, \infty)}\right)^{(0)}$.

We will use the inequalities satisfied by the singular values.

$$
\mu_{n+m}(A B) \leq \mu_{n}(A) \mu_{m}(B)
$$

Choose $n+m=2 N+j$ where $j=0,1$ and look at the following limit,

$$
\limsup _{N \rightarrow \infty}(2 N+j) \mu_{2 N+j}(A B) \leq \limsup _{N \rightarrow \infty}(2 N+j)^{1 / 2} \mu_{N}(A)(2 N+j)^{1 / 2} \mu_{N+j}(B) .
$$

\footnotetext{
${ }^{16}$ Unfortunately, we could not incorporate his suggestions into this version.

${ }^{17}$ The symbol $s_{n}=O\left(\pi_{n}\right)$ means that, $\lim \sup _{n \rightarrow \infty} \frac{s_{n}}{\pi_{n}}<\infty$ and $s_{n}=o\left(\pi_{n}\right)$ iff $\lim _{n \rightarrow \infty} \frac{s_{n}}{\pi_{n}}=0$.
} 
Now we can use,

$$
\lim _{N \rightarrow \infty}\left(N^{1 / 2} \mu_{N}(A)\right)=a<\infty \text { and } \lim _{N \rightarrow \infty}\left(\left(N^{1 / 2} \mu_{N}(B)\right)=0\right.
$$

in the above expression to get,

$$
\lim _{N \rightarrow \infty}(2 N+j) \mu_{2 N+j}(A B)=0
$$

and this implies that the product is in the closure, $A B \in\left(\mathcal{L}^{(1, \infty)}\right)^{(0)}$. One can imitate the above proof to show that $A, B \in \mathcal{L}^{(2, \infty)}$ then $A B \in \mathcal{L}^{(1, \infty)}$; we leave this to the reader.

Let us use the same idea to show that $K(\mathcal{H}) \mathcal{L}^{(2, \infty)} \subset\left(\mathcal{L}^{(2, \infty)}\right)^{(0)}$;

$$
\limsup _{2 N+j}(2 N+j)^{1 / 2} \mu_{2 N+j}(A K) \leq \limsup _{2 N+j}(2 N+j)^{1 / 2} \mu_{N}(A) \mu_{N+j}(K)=0,
$$

by using the fact that $\lim _{N} \mu_{N}(K)=0$ for a compact operator $K$.

In the second part we will prove some of the properties of the conditional Dixmier traces, which are identical to the usual trace conditions. We define the conditional trace as

$$
\operatorname{Tr}_{\omega}^{\epsilon} A=\operatorname{Tr}_{\omega}^{\epsilon}\left(\begin{array}{ll}
a_{11} & a_{12} \\
a_{21} & a_{22}
\end{array}\right)=\frac{1}{2}\left(\operatorname{Tr}_{\omega}\left(a_{11}\right)+\operatorname{Tr}_{\omega}\left(a_{22}\right)\right)=\frac{1}{4} \operatorname{Tr}_{\omega}[A+\epsilon A \epsilon] .
$$

for $A=\left(\begin{array}{ll}a_{11} & a_{12} \\ a_{21} & a_{22}\end{array}\right)$. Notice that we have absorbed a factor of $1 / 2$ into the definition to make the formulae involving this trace look simpler. First property;

$$
\operatorname{Tr}_{\omega}^{\epsilon} A B=\operatorname{Tr}_{\omega}^{\epsilon} B A
$$

if all the individual terms in the products $\sum_{k} a_{i k} b_{k i}$ are in the ideal $\mathcal{L}^{(1, \infty)}$. This is easy to see if we use the definition of the conditional trace, and,

$$
\left(\begin{array}{ll}
a_{11} & a_{12} \\
a_{21} & a_{22}
\end{array}\right)\left(\begin{array}{ll}
b_{11} & b_{12} \\
b_{21} & b_{22}
\end{array}\right)=\left(\begin{array}{cc}
a_{11} b_{11}+a_{12} b_{21} & * \\
* & a_{21} b_{12}+a_{22} b_{22}
\end{array}\right)
$$

If each of the terms appearing in the diagonal parts are indepently in $\mathcal{L}^{(1, \infty)}$, we can use $\operatorname{Tr}_{\omega} a b=\operatorname{Tr}_{\omega} b a$ and see that the result is the same when one takes the product in the opposite order.

We will show that the group representation is satisfied upto a central term. It is more convenient to use $\operatorname{det}_{\omega}$ instead of $e^{\operatorname{Tr}_{\omega}}$ and the power $1 / \hbar$ is not written since it is easy to put back. We compare the two ways of applying the representation, $\rho\left(g_{2}^{-1}\right) \rho\left(g_{1}^{-1}\right) \Psi(Z)$ and $\rho\left(\left(g_{1} g_{2}\right)^{-1}\right) \Psi(Z)$. The first expression gives,

$$
\operatorname{det}_{\omega}\left(d_{1}^{-1} c_{1}\left(a_{2} Z+b_{2}\right)\left(c_{2} Z+d_{2}\right)^{-1}+1\right) \operatorname{det}_{\omega}\left(d_{2}^{-1} c_{2} Z+1\right) \Psi\left(\left(g_{1} g_{2}\right) \circ Z\right)
$$

and the second

$$
\operatorname{det}_{\omega}\left(\left(d_{1} d_{2}+c_{1} b_{2}\right)^{-1}\left(c_{1} a_{2}+d_{1} c_{2}\right) Z+1\right) \Psi\left(\left(g_{1} g_{2}\right)^{-1} \circ Z\right)
$$


It is enough to compare the "determinant" pieces because the other parts are the same. Let us check the following,

$$
\begin{aligned}
\operatorname{det}_{\omega} & \left(d_{1}^{-1} c_{1}\left(a_{2} Z+b_{2}\right)\left(c_{2} Z+d_{2}\right)^{-1}+1\right) \operatorname{det}_{\omega}\left(d_{2}^{-1} c_{2} Z+1\right) \\
= & \operatorname{det}_{\omega}\left(d_{2}^{-1} d_{1}^{-1} c_{1}\left(a_{2} Z+b_{2}\right)\left(d_{2}^{-1} c_{2} Z+1\right)^{-1}+1\right) \operatorname{det}_{\omega}\left(d_{2}^{-1} c_{2} Z+1\right) \\
= & \operatorname{det}_{\omega}\left(\left(d_{1} d_{2}\right)^{-1} c_{1}\left(a_{2} Z+b_{2}\right)\left(d_{2}^{-1} c_{2} Z+1\right)^{-1}\left(d_{2}^{-1} c_{2} Z+1\right)+d_{2}^{-1} c_{2} Z+1\right) \\
= & \operatorname{det}_{\omega}\left(\left(d_{1} d_{2}\right)^{-1} c_{1}\left(a_{2} Z+b_{2}\right)+d_{2}^{-1} c_{2} Z+1\right) \\
= & \operatorname{det}_{\omega}\left(\left(d_{1} d_{2}\right)^{-1}\left(c_{1} a_{2}+d_{1} c_{2}\right) Z+\left(d_{1} d_{2}\right)^{-1} c_{1} b_{2}+1\right) .
\end{aligned}
$$

We used the multiplicative property of the $\operatorname{det}_{\omega}$, which comes from the properties of the Dixmier trace. The equalities are true by adding terms which give zero under dixmier trace, this is the advantage of using the symbol $\operatorname{det}_{\omega}$. let us compare this with

$$
\begin{aligned}
\operatorname{det}_{\omega} & \left(\left(d_{1} d_{2}+c_{1} b_{2}\right)^{-1}\left(c_{1} a_{2}+d_{1} c_{2}\right) Z+1\right)= \\
= & \operatorname{det}_{\omega}\left[\left(\left(d_{1} d_{2}\right)^{-1} c_{1} b_{2}+1\right)^{-1}\left(d_{1} d_{2}\right)^{-1}\left(c_{1} a_{2}+d_{1} c_{2}\right) Z+1\right) \\
= & \operatorname{det}_{\omega}\left(\left(d_{1} d_{2}\right)^{-1} c_{1} b_{2}+1\right)^{-1} \operatorname{det}_{\omega}\left(\left(d_{1} d_{2}\right)^{-1}\left(c_{1} a_{2}+d_{1} c_{2}\right) Z+\left(d_{1} d_{2}\right)^{-1} c_{1} b_{2}+1\right)
\end{aligned}
$$

hence they differ by a constant multiple, which never vanihes,

$$
c_{\omega}\left(g_{1}, g_{2}\right)=\operatorname{det}_{\omega}\left(\left(d_{1} d_{2}\right)^{-1} c_{1} b_{2}+1\right)
$$

This trace is well-defined as one can check and since it never vanishes the two sided are equal. We need to further check that it obeys the cocyle condition,

$$
\begin{aligned}
c_{\omega}\left(g_{1} g_{2}, g_{3}\right) c_{\omega}\left(g_{1}, g_{2}\right) & =c_{\omega}\left(g_{1}, g_{2} g_{3}\right) c_{\omega}\left(g_{2}, g_{3}\right) \\
& =\operatorname{det}^{\frac{1}{\hbar}}\left[\left(d_{1} d_{2} d_{3}\right)^{-1} c_{1} a_{2} b_{3}+\left(d_{2} d_{3}\right)^{-1} c_{2} b_{3}+\left(d_{1} d_{2}\right)^{-1} c_{1} b_{2}+1\right] .
\end{aligned}
$$

The sum of all the terms except 1 inside the determinant sign are in the ideal $\mathcal{L}^{(1, \infty)}$, hence the Dixmier trace is well defined. The cocycle $c_{\omega}$, in the finite dimensional case, can be obtained from $\phi(g)=\operatorname{det}(d)$, as $c\left(g_{1}, g_{2}\right)=\phi\left(g_{1}\right) \phi\left(g_{2}\right) \phi\left(g_{1} g_{2}\right)^{-1}$. Clearly it is not well-defined in infinite dimensions; in fact, the extension is nontrivial. Thus, we obtain a representation of a central extension $\hat{U}_{1+}\left(\mathcal{H}_{-}, \mathcal{H}_{+}\right)$in the Quantum Hilbert space of holomorphic sections.

\section{$7 \quad$ small appendix}

This appendix does not belong to the actual paper, since we could not find a reference for the direct proof of the claim in the footnote, we include it for those who are interested in. It is certainly known and implicit ( or stated in a more general context) in the paper of Connes "non-commutative geometry" IHES Publ. Math. vol. 62, pg 257.

Here we will give the proof that the general linear group is a topological product of unitary times positive hermitian elements: 
We remind the reader the following formula for the square root of a positive element:

$$
A^{1 / 2}=\frac{1}{\pi} \int_{0}^{\infty} \frac{d \lambda}{\lambda^{1 / 2}} A(\lambda I+A)^{-1}
$$

We need to show that under the new norm $\left\|[\epsilon, A]_{+}\right\|+\|[\epsilon, A]\|_{\mathcal{L}^{(2, \infty)}}=\|a\|+\|d\|+\|b\|_{\mathcal{L}^{(2, \infty)}}+$ $\|c\|_{\mathcal{L}^{(2, \infty)}}$, the operation is well defined and continuous. First let us show that it is welldefined. we can compute first;

$$
\left\|\left[\epsilon, A^{1 / 2}\right]\right\|_{\mathcal{L}^{(2, \infty)}} \leq \frac{1}{\pi} \int_{0}^{\infty} d \lambda \lambda^{1 / 2}\left\|\left[\epsilon,(\lambda I+A)^{-1}\right]\right\|_{\mathcal{L}^{(2, \infty)}}
$$

using $A(\lambda I+A)^{-1}=1-\lambda(\lambda I+A)^{-1}$. We can use now, $\left[\epsilon,(\lambda I+A)^{-1}\right]=-(\lambda I+A)^{-1}[\epsilon, A](\lambda I+$ $A)^{-1}$, and the symmetric norm property;

$$
\begin{aligned}
\left\|\left[\epsilon, A^{1 / 2}\right]\right\|_{\mathcal{L}^{(2, \infty)}} & \leq \frac{1}{\pi} \int_{0}^{\infty} d \lambda \lambda^{1 / 2}\left\|(\lambda I+A)^{-1}\right\|^{2}\|[\epsilon, A]\|_{\mathcal{L}^{(2, \infty)}} \\
& \leq \frac{1}{\pi} \int_{0}^{\infty} d \lambda \lambda^{1 / 2} \frac{1}{(\lambda+\inf \sigma(A))^{2}}\|[\epsilon, A]\|_{\mathcal{L}^{(2, \infty)}}
\end{aligned}
$$

Here we used the fact that the spectrum satisfies $\sigma\left((\lambda I+A)^{-1}\right)=(\sigma(\lambda I+A))^{-1}$. This shows that the spectrum is contained in $\left[(\lambda+\|A\|)^{-1},(\lambda+\inf \sigma(A))^{-1}\right]$. The infimum of the spectrum of $A$, is a positive number; hence the integral on the right is actually convergent in the $\lambda \approx 0$ region, and as for the $\lambda \rightarrow \infty$, it is clear that the integral is dominated by the integral of $\lambda^{-3 / 2}$, hence convergent. So we have a finite number times the norm of the off-diagonal parts, which are finite. For the diagonal parts we know that the square root is a bounded operator [4, and we use $\left\|P_{+} B P_{+}\right\| \leq\|B\|$, same for $\left\|P_{-} B P_{-}\right\|$, for any bounded operator $B$, where $P_{+}, P_{-}$are projections onto $\mathcal{H}_{+}, \mathcal{H}_{-}$respectively. This shows that each one is bounded.

For the continuity, we use similar ideas; first prove this for bounded operators:

$$
\begin{aligned}
\left\|A^{1 / 2}-B^{1 / 2}\right\| & \leq \frac{1}{\pi} \int_{0}^{\infty} d \lambda \lambda^{1 / 2}\left\|(\lambda I+A)^{-1}-(\lambda I+B)^{-1}\right\| \\
& \leq \frac{2}{\pi} \int_{0}^{\infty} d \lambda \lambda^{1 / 2}\left\|(\lambda I+A)^{-1}\right\|^{2}\|A-B\| \leq C\|A-B\| .
\end{aligned}
$$

Now we can apply a similar method for the off-daigonal parts;

$$
\begin{aligned}
\left\|\left[\epsilon, A^{1 / 2}-B^{1 / 2}\right]\right\|_{\mathcal{L}^{(2, \infty)}} & \leq \\
\frac{1}{\pi} \int_{0}^{\infty} & d \lambda \lambda^{1 / 2} 2\left\|(\lambda I+A)^{-1}[\epsilon, A](\lambda I+A)^{-1}-(\lambda I+B)^{-1}[\epsilon, B](\lambda I+B)^{-1}\right\|_{\mathcal{L}^{(2, \infty)}} \\
& \leq \frac{1}{\pi} \int_{0}^{\infty} d \lambda \lambda^{1 / 2}\left(\left\|(\lambda I+B)^{-1}\right\|^{2}\|[\epsilon, A-B]\|_{\mathcal{L}^{(2, \infty)}}\right. \\
& \left.+2\left\|(\lambda I+B)^{-1}\right\|\left\|^{3}\right\|[\epsilon, A]\left\|_{\mathcal{L}^{(2, \infty)}}\right\| A-B \|\right)
\end{aligned}
$$

in a small neighborhood of $A-B$-that is for $\left\|[\epsilon, A-B]_{+}\right\|+\|[\epsilon, A-B]\|_{\mathcal{L}^{(2, \infty)}}$ sufficiently small. All the integrals are convergent and the continuity is clear from this. SInce $A \mapsto A^{\dagger} A$ 
is continous under the same topology, the polar decomposition is a continous operation in this ideal. We remark that the above proof remains valid for any symmetrically normed ideal.

Let us also comment on the proof that $G L_{1+}$ is contractible to its unitary subgroup. Define the polar decomposition, $A=U_{A}|A|$, where $|A|=\left(A^{\dagger} A\right)^{1 / 2}$. We have shown that the absolute value map is continuous in the topology we use. Then we can obtain a homotopy to the unitary part, by using

$$
A(t)=U_{A} \exp (t \log |A|) .
$$

This gives $A(1)=A$ and $A(0)=U_{A}$. Of course we need to justify that the above functions are all continuous in the product topology. This can be done by using the following integral representation for $\log A$ for positive $A$;

$$
\log A=(A-I) \int_{0}^{1} d s((1-s) I+s A)^{-1}
$$

One can give a self-contained proof by using a very similar idea as in the first case. For this we need to employ a similar identity for the fractional powers of positive operators:

$$
A^{\alpha}=\frac{\sin \alpha \pi}{\pi} \int_{0}^{\infty} d \lambda \lambda^{\alpha-1}(\lambda 1+A)^{-1} A,
$$

for $0<\alpha<1$. By using exactly the same types of ideas we can prove that the map $G L_{1+}(\mathcal{H}) \times[0,1] \rightarrow U_{1+}(\mathcal{H})$ is continuous, hence $U_{1+}$ is a deformation retract of $G L_{1+}$. We give a sketch here. Let us estimate the norm of $A^{\alpha}$;

$$
\left\|A^{\alpha}\right\| \leq \frac{|\sin \alpha \pi|}{\pi} \int_{0}^{\infty} d \lambda \lambda^{\alpha-1}\|A\|(\lambda+\|A\|)^{-1}
$$

which is finite for $\alpha \in(0,1)$. First we show that $\left\|A^{\alpha}-B^{\beta}\right\|$ can be made arbitrarily small by choosing $\left\|[\epsilon, A-B]_{+}\right\|+\|[\epsilon, A-B]\|_{\mathcal{L}^{(2, \infty)}}$ and $|\alpha-\beta|$ sufficiently small.

$$
\begin{aligned}
\| A^{\alpha} & -B^{\beta}\left\|\leq \frac{|\sin \alpha \pi|}{\pi} \int_{0}^{\infty} d \lambda \lambda^{\alpha} 4\right\|(\lambda I+B)^{-1}\left\|^{2}\right\| A-B \| \\
& +\left|\frac{\sin \alpha \pi}{\pi}-\frac{\sin \beta \pi}{\pi}\right| \int_{0}^{\infty} d \lambda \lambda^{\alpha-1}|| B\left\|(\lambda+|| B \|)^{-1}+\frac{|\sin \beta \pi|}{\pi} \mid \int_{0}^{\infty}\left(\lambda^{\alpha-1}-\lambda^{\beta-1}\right)\right\| B \|(\lambda+\|B\|)^{-1}
\end{aligned}
$$

One can see that all the terms above can be made as small as we wish. This shows that the diagonal elements satisfy the required continuity. For the off-diagonal parts;

$$
\begin{aligned}
& \left\|\left[\epsilon, A^{\alpha}-B^{\beta}\right]\right\|_{\mathcal{L}^{(2, \infty)}} \leq \frac{|\sin \alpha \pi|}{\pi} \int_{0}^{\infty} d \lambda \lambda^{\alpha}\left\|(\lambda I+B)^{-1}\right\|^{2}\left(\|[\epsilon, A-B]\|_{\mathcal{L}^{(2, \infty)}}+4\|A-B\|\|[\epsilon, B]\| \|_{\mathcal{L}^{(2, \infty)}}\right) \\
& \quad+\left|\frac{\sin \alpha \pi}{\pi}-\frac{\sin \beta \pi}{\pi}\right| \int_{0}^{\infty} d \lambda \lambda^{\alpha-1}||(\lambda I+B)^{-1}\|\|[\epsilon, B] \|_{\mathcal{L}^{(2, \infty)}}\left(\|B\|+\left\|(\lambda I+B)^{-1}\right\|\right) \\
& \quad+\frac{|\sin \alpha \pi|}{\pi}\left|\int _ { 0 } ^ { \infty } d \lambda ( \lambda ^ { \alpha } - \lambda ^ { \beta } ) \left\|(\lambda I+B)^{-1}\left|\left\|^{2}\right\|[\epsilon, B] \|_{\mathcal{L}^{(2, \infty)}}\right|\right.\right.
\end{aligned}
$$

where all the terms can be made arbitrarily small by choosing a small enough neighborhood. Thus we prove the joint continuity.

Incidentally we remark that the proof does not depend on the specific ideal. 


\section{References}

[1] R. Abraham and J. E. Marsden, Foundations of Mechanics, 2nd Ed., the Benjamin/Cummings Publ. Comp., Inc. 1978.

[2] V. I. Arnold, Mathematical Methods of Classical Mechanics, 2nd Ed., Springer Verlag, New York, 1986.

[3] S. Axelrod, S. D. Pietra, and E. Witten, Jour. Diff. Geom. 33 (1991) 787.

[4] O. Bratelli and D. Robinson, Operator Algebras in Physics, Vol. I, Springer verlag, 1985.

[5] F. A. Berezin, Comm. Math. Phys. 40 (1975) 153.

[6] A. Connes, Comm. Math. Phys. 117 (1988) 673.

[7] A. Connes, Non-commutative geometry; spectral aspects, les houches lectures, 1995. Non-commutative Geometry, Academic Press, 1994.

[8] K. J. Dykema, T. Figiel, G. Weiss, and M. Wodzicki, "the commutator structure of operator ideals", preprint.

[9] E. Langmann, Comm. math. Phys. 162 (1994) 1.

[10] M. Cederwall, G. Ferretti, B. E. W. Nilsson and A. Westerberg, Comm. Math. Phys. 175 (1996) 203

[11] B. Fedosov, F. Golse, E. Leichtnam, and E. Shrohe, Journ. Funct. Analysis, 142 (1996) 1.

[12] I. C. Gohberg and M. G. Krein,Intorduction to The Theory of Nonself-adjoint Linear Operators, Trans. Am. math. Soc. 1968.

[13] N. E. Hurt, Geometric Quantization in Action, D. Reidel, Dordrecht, 1983.

[14] A. Kirillov, Geometric Quantization, in Dynamical Systems IV, ed. by V. I.. Arnold, Springer Verlag, 1988.

[15] M. Kontsevich and S. Vishik, Conference proceeding, hep-th 9405035.

[16] Mickelsson, Talk in baltic school in field theory, hep-th 9404093, E. Langmann and J. Mickelsson, Lett. math. Phys. 36 (1996) 45

[17] J. Mickelsson and S. G. Rajeev, Comm. Math. Phys. 116 (1988) 365, J. Mickelsson, Comm. Math. Phys. 154 (1993) 403.

[18] A. Pressley and G. Segal, Loop Groups, Oxford University press, 1988 
[19] D. Pickrell, Journ. Funct. Anal. 70 (1987) 323.

[20] S. G. Rajeev, Int. J. Mod. Phys.A 9 (1994) 5583

[21] S. G. Rajeev and O.T.Turgut, Comm. Math. Phys. 192 (1998) pg 493.

[22] B. Simon,Trace Ideals and Their Applications, Cambridge Univ. Press, Cambridge, 1979.

[23] Wodzicki, Invent. Math. 75, 143 (1984) 